\title{
Marco jurídico de las pensiones mexicanas: análisis empírico mediante productos financieros "estructurados" en el periodo 2018-2019
}

\section{Legal framework of Mexican pensions: Empirical analysis through structured financial products in the period 2018-2019}

\author{
Héctor Alonso Olivares-Aguayo, Ana Laura Medina-Conde \\ y Oscar Javier Apáez-Pineda
}

Facultad de Negocios de la Universidad La Salle-México, Universidad

Autónoma de Tlaxcala, México, Facultad de Derecho de la Universidad

La Salle-México

Resumen

La ley establece que en las Administradoras de Fondos para el Retiro (AFORES) el dinero de los ahorradores del sistema de pensiones es invertido, buscando obtener el mayor rendimiento posible, sin embargo, actualmente dichos rendimientos pueden ser negativos, por ello, el objetivo general de la investigación propone como alternativa una total inversión de los recursos para cada Sociedad de Inversión Especializada en Fondos para el Retiro (SIEFORE) en productos financieros "estructurados" Put Spread y Call Spread considerando como subyacentes los principales índices sectoriales invertibles (Materiales, Industrial, Servicios Financieros y Consumo Frecuente). Los resultados muestran que invertir en este tipo de productos los recursos que se tienen en cada tipo de SIEFORE, las ganancias obtenidas en el Sector Materiales son mejores a las presentadas por la Comisión Nacional del Sistema de Ahorro para el Retiro (CONSAR) al cierre del primer y segundo trimestre de 2019, y para los demás sectores, los rendimientos siempre se muestran positivos.

Palabras clave: AFORE, derecho laboral, seguridad social, pensiones.

Abstract

The law establishes that in retirement management fund (AFORES) the money of the pension system savers is invested, seeking to obtain the highest possible returns, however, currently these returns can be negative, therefore, the general objective of the research propose as an alternative the total investment of the resources for each SIEFORE in structured financial products Put Spread and Call Spread considering the main invertible sector indices (Materials, Industrial, Financial Services and Consumer Products) as subyacent. The results show that by investing in this type of products the resources that are available in each type of SIEFORE, the profits obtained in the Materials Sector are better than those presented by CONSAR at the end of the first and second quarter of 2019, and for the other sectors, the returns was always positive.

Keywords: AFORE, labor law, social security, pensions. 


\section{INTRODUCCIÓN}

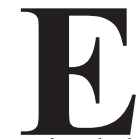

1 sistema chileno de pensiones fue pionero en la implementación de fórmulas de capitalización individual y su modelo fue exportado a muchos países, entre ellos México, en donde a partir de la mitad de la década de los noventa se implementó para los trabajadores de la iniciativa privada.

Actualmente a decir de Lara y Silva (2014); Pérez et al. (2017); Aguiló y Echeverría (2020) el sistema de pensiones chileno ha sufrido críticas mundiales, pues una vez que ha comenzado a pensionar a sus trabajadores, las pensiones obtenidas no lograron superar el salario mínimo vigente en la sociedad chilena, aunado a que el mismo es insuficiente para los pensionados ante el costo de vida actual.

El presente trabajo de investigación parte de la hipótesis de que incrementar el porcentaje de inversión en "estructurados" mejorará las ganancias obtenidas por los recursos destinados en las Sociedad de Inversión Especializada en Fondos para el Retiro (SIEFORES), lo que permitiría cumplir con la disposición legal de invertir el dinero de los ahorradores del sistema de pensiones buscando obtener el mayor rendimiento posible.

Los objetivos particulares de la investigación son: Analizar los antecedentes históricos de la reforma al sistema de pensiones de los trabajadores regulados por el Apartado A del Artículo 123 constitucional, es decir, los trabajadores de la iniciativa privada, apartado en el que además se analizan los argumentos y promesas utilizadas para sustentarla.

Precisar las normas jurídicas que se ven relacionadas con el sistema de las Administradoras de Fondos para el Retiro (AFORES), ${ }^{1}$ presentando un panorama general de la normatividad positiva vigente, sus interrelaciones y las interpretaciones que en relación con ella ha emitido la Corte.

Proponer con base en un análisis jurídico y financiero las medidas que deberían implementarse con la finalidad de que los rendimientos pudieran obtener mejores frutos y se precisen las acciones legales y financieras necesarias para tal efecto como es la propuesta de inversiones en productos financieros "estructurados" Put Spread y Call Spread.

\footnotetext{
${ }^{1}$ El artículo 18 de la Ley del SAR define a las AFORES como aquellas que de manera habitual y profesional se dedican a administrar las cuentas individuales en su carácter de entidades financieras, así como gestionar Sociedades de Inversión (LSAR 2014). Se crean como Sociedades Anónimas de Capital Variable y son autorizadas por la Comisión Nacional del Sistema de Ahorro para el Retiro (CONSAR), por el manejo del ahorro se cobran comisiones, a través de las SIEFORES que es un fondo de inversión en el cual las AFORES funcionan como un mecanismo de inversión de los recursos de los trabajadores, existen cuatro SIEFORES básicas que dependen directamente de la edad y el riesgo de vejez disminuye cuando ésta aumenta.
} 
La estructura del documento inicia con una parte por precisar el origen de las AFORES en México, los antecedentes del Sistema de Ahorro para el Retiro (SAR) y continua con la precisión de lo que son las notas Estructuradas Call Spread y Put Spread; y el análisis de los resultados; por último, se presentan las conclusiones y reflexiones finales producto de la investigación.

\section{El ORIGEN DE LAS AFORES}

El sistema de las Administradoras de Fondos para el Retiro (AFORES) fue implementado en México a mediados de la década de los noventas, a través de una reforma al sistema de pensiones del régimen laboral de los trabajadores, mismos que de conformidad con la Constitución Política de los Estados Unidos Mexicanos (CPEUM) en su artículo 123 apartado A y de sus leyes reglamentarias (Ley Federal del Trabajo y la Ley del Seguro Social) establecen el aseguramiento y protección del riesgo de vejez en el Instituto Mexicano del Seguro Social (IMSS), institución que a su vez se encuentra regulada por su Ley respectiva, por lo que las pensiones son parte integrante de la Seguridad Social de las personas.

Uno de los antecedentes más antiguos de la Seguridad Social es la caridad, el problema de este tipo de ayuda es que dependía de la buena voluntad de las personas y no existía seguridad jurídica. Setter (1982) y De Buen (1997) dicen que es en Alemania en 1883 donde surgen los primeros regímenes contra la enfermedad y la vejez. Siendo el Canciller Otto Von Bismarck, quien le da carácter tripartito y surge el primer Código de Seguridad Social.

En México Ignacio Ramírez intentó incorporar en la Constitución de 1857 la protección a huérfanos, mujeres y trabajadores, pero fue hasta la Constitución de 1917 que se instaura ese derecho.

El concepto de Seguridad Social, a decir de Ruiz (1999), surge en 1994 cuando la Organización Internacional del Trabajo (OIT) realiza un intento por definir a la Seguridad Social aún como una medida que la sociedad establece para sus miembros contra los riesgos que con sus recursos propios no puede cubrir, Beveridge como se citó en Olea (1983) concuerda con Arce (1972) en que corresponde al Estado establecer medidas para proteger al ciudadano en situación de necesidad, como en la vejez. Sainz (2008) coincide con Sánchez (1987) en que la Seguridad Social es una forma de protección de los trabajadores en su relación de subordinación. Sin embargo, existe un pacto social en el que el ciudadano entrega al Estado a través de las contribuciones una parte de sus ingresos para que este lo regrese en 
bienes y en servicios, por lo que la Seguridad Social no es un acto de caridad del Estado sino un Derecho, una contraprestación bilateral.

La OIT (2001) en la definición de Seguridad Social incorpora elementos como asegurar la asistencia médica y el ingreso en: la vejez, maternidad, invalidez y accidentes de trabajo.

El fundamento constitucional de la Seguridad Social se encuentra en la fracción XXIX del Artículo 123, que establece como utilidad social el establecimiento de cajas de seguro populares de: invalidez, vida, cesación involuntaria del trabajo, accidentes en el que tanto el gobierno federal como el de cada estado deben fomentar la organización de instituciones de esta índole (CPEUM, 2019).

Las principales instituciones de Seguridad Social en el país son el Instituto Mexicano del Seguro Social (IMSS), el Instituto de Seguridad y Servicios Sociales de los Trabajadores del Estado (ISSSTE) y el Instituto del Fondo Nacional de la Vivienda para los Trabajadores (INFONAVIT) dentro de estos existen regímenes específicos de protección de riegos. En el IMSS que es un organismo público descentralizado con personalidad jurídica y patrimonio propio de naturaleza tripartita y donde se encuentra el régimen obligatorio, se establece la contingencia del Retiro de Cesantía en Edad Avanzada y Vejez donde las condiciones para tener derecho a una pensión futura son: la edad de 60 hasta 65 años y haber cotizado ante el IMSS un mínimo de 1250 semanas (aproximadamente 24 años).

La Ley del Seguro Social (LSS) fue reformada en diciembre de 1995 antes de esta ley solo eran necesarias 500 cotizaciones, (Solís, 2001; Ruiz-Medina, Quintero y Cuadras, 2016; García, 2015) afirman que se operaba a través de un sistema de reparto que era administrado por el IMSS, las pensiones se pagaban con los aportes de los trabajadores, los patrones y el gobierno, más los rendimientos de los fondos de retiro acumulados, entre las causas en que se fundamenta la reforma se encuentran: cambios demográficos (aumento en la esperanza de vida), la inviabilidad financiera y eventos económicos desfavorables. Lo anterior aunado a que se pronosticaban eventos optimistas de incrementar el ahorro por varias vías.

El primero de julio de 1997 se transita la administración a cuentas individuales administradas por las AFORES. Sin embargo, a decir de la OIT (2018) entre 1981 y 2014, treinta países privatizaron total o parcialmente su sistema público de pensiones obligatorias, entre ellos México impulsados por el Banco Mundial (BM), el Fondo Monetario Internacional (FMI), la Organización para la Cooperación y el Desarrollo Económico (OCDE), entre otros organismos, y que para 2018; dieciocho países revirtieron estas 
reformas cuando se analizaron los inconvenientes de la administración del sector privado.

Cruz (1997) afirma que con la reforma se deja al mercado la determinación del monto de las pensiones y tiene importancia para las instituciones de Seguridad Social; pues, se traslada a entidades financieras privadas el manejo de cuantiosos recursos fiscales.

\section{Antecedentes del Sistema de Ahorro para el Retiro (SAR)}

Uno de los argumentos principales para la reforma del sistema de pensiones fue el desequilibrio actuarial que comenzó a notarse en el sistema de reparto (Nava y Ham, 2006), pero previo a su modificación se creó el Sistema de Ahorro para el Retiro (SAR-92).

En 1992, el presidente Carlos Salinas de Gortari echó a andar el SAR, este sistema funcionó con las aportaciones que realizaban los patrones para el retiro de los trabajadores y las del fondo de vivienda. Estos recursos se depositaron en bancos, quienes, con un pago de comisión, los enviaron al Banco de México, para que este último los invirtiera en bonos de deuda pública.

Aunado a ello, en 1994 se creó la Comisión Nacional del Sistema de Ahorro para el Retiro (CONSAR) como un órgano desconcentrado de la Secretaria de Hacienda y Crédito Público (SHCP) y concentrador con facultades de regulación (control y vigilancia del SAR 92). De conformidad con la ley la CONSAR es desde ese entonces la encargada de vigilar el funcionamiento de los Sistemas de Ahorro para el Retiro.

Para 1995, se lleva a cabo la reforma a la Ley del Seguro Social, misma que tuvo como objetivo cambiar el régimen de pensiones que se sustentaba en el sistema de reparto con beneficio definido, bajo el principio de solidaridad generacional. La propuesta fue un régimen de cuentas individualizadas por lo que la reforma trajo consigo la creación del nuevo sistema de pensiones por capitalización.

Esta ley fue preparada durante el gobierno del presidente Carlos Salinas, pero publicada por el gobierno de Ernesto Zedillo y sus principales cambios fueron que se abandonó el sistema de pensiones solidario, o de reparto, para dar pie a uno de cuentas individualizadas, o de capitalización; estas cuentas quedaron entonces en manos de intermediarios financieros, pertenecientes a los bancos denominados AFORES.

Para completar la reforma y que el sistema pudiera funcionar en 1996 se publicó en el Diario Oficial de la Federación la Ley del SAR; así como reformas a diversas legislaciones relacionadas, en ella, se dio un papel 
preponderante a la CONSAR como el órgano regulador; y se legisló en relación con la implementación del sistema de AFORES y las denominadas SIEFORES; que son las que directamente invierten los recursos de los trabajadores principalmente en el mercado de valores.

El sistema comienza a funcionar a partir del $1^{\circ}$ de julio de 1997, y para ese entonces ya se habían modificado diversos ordenamientos, asimismo, se comenzó a precisar el trabajo de las SIEFORES como entidades financieras administradas y operadas por las AFORES y autorizadas por la CONSAR.

Esto tenía como objetivo invertir el ahorro de las cuentas individuales, de manera independiente a las AFORES a través de diversas SIEFORES básicas según el rango de edad de los trabajadores, pues, la ley estableció que los recursos de las cuentas individuales se invirtieran en la SIEFORE correspondiente a cada trabajador, según su edad.

En atención con lo anterior el rango de edad de los trabajadores, produjo cuatro tipos de SIEFORES que son las siguientes: SB1 (60 años o mayores), SB2 (46 a 59 años), SB3 (37 a 45 años) y SB4 (36 años o menores).

Antes de la reforma de 2009 a la Ley del SAR las comisiones llegaban a 1.87 por ciento ahora se encuentran en promedio en 0.98 por ciento, ya que, la Junta de Gobierno adquiere facultades para autorizar dichas comisiones.

La Tesis jurisprudencial 2a./J. 26/2011 afirma respecto de las comisiones que "El artículo 37 de la ley relativa que prevé el procedimiento al que deben ajustarse las AFORES para determinar las comisiones que podrán cobrar, no viola el artículo 39 de la constitución federal" (legislación vigente a partir del 22 de enero de 2009).

El artículo 37 de la Ley del SAR, al establecer el procedimiento al que deben ajustarse las AFORES para determinar las comisiones que por sus servicios podrán cobrar a los trabajadores por el manejo económico de los fondos de las cuentas individuales de ahorro para el retiro bajo su encargo, no viola el principio de poder público dimanado del pueblo soberano, contenido en el artículo 39 de la Constitución Federal, porque el hecho de que la comisión sea con cargo a la totalidad de los activos administrados no llega al extremo de provocar que los poderes públicos dejen de desempeñarse en beneficio del pueblo, en tanto que, inclusive, la sola existencia de la regulación en la materia (ámbito legislativo), su aplicación a los destinatarios por parte de las autoridades respectivas (ámbito ejecutivo) y su análisis a través de los medios de defensa procedentes (ámbito judicial), pone de manifiesto que los poderes públicos están abocados en su respectiva esfera 
competencial a la atención de la temática inherente al (SAR) en beneficio de la población y, de manera particular, de su sector laboral, en la vertiente relativa a la Seguridad Social.

La CONSAR (2019a) afirma que la comisión de uno por ciento sobre el saldo de la cuenta de ahorro acumulado puede reducir la pensión en un periodo de 40 años en 20 por ciento de acuerdo a un análisis de la OCDE.

El 31 de mayo de 2019 se publicaron diversas modificaciones al Régimen de Inversión de las SIEFORES, en el que se cambia el esquema de multifondos a uno de fondos generacionales llamados Fondos de Inversión Especializada en Fondos para el Retiro (Fiefores), como una estrategia de inversión de largo plazo. Estos fondos invitan a que las inversiones puedan realizarse en productos financieros derivados.

Algunas investigaciones recientes sobre los planes de pensiones a nivel mundial se pueden apreciar en Amaro y Alfonso (2018), quienes analizan los impactos del envejecimiento de la población en los planes de pensiones de reparto en tres países: Brasil, España y Francia. Por su parte Koh et al. (2021) se basan en el panel de vida de Singapur evaluando cómo la confianza se vincula con la participación y los retiros del plan de pensiones de los adultos mayores. Kim et al. (2021) estudian el caso para Estados Unidos examinando la exclusión del costo de las pensiones de los gastos salariales en el sector público utilizando un panel de datos de 15 años de planes estatales de pensiones para maestros.

Para el caso de México algunas investigaciones interesantes se pueden consultar en Farfán (2017) que analiza cómo la Constitución de 1917, artículo 123, fracción XXIX, así como la normativa correspondiente, definen los fundamentos del modelo de seguridad social mexicano. Murillo y Venegas (2011) obtienen estimaciones de indicadores de cobertura previsional o retiro jubilatorio para la población mexicana mayor de 65 años, y evalúan el impacto de los sistemas de pensiones en las transiciones hacia la jubilación de adultos en edades medias y avanzadas, utilizan datos microeconómicos de la Encuesta Nacional de Salud y Envejecimiento de México. Por su parte Rodríguez (2017) analiza el riesgo de longevidad, definido como la posibilidad de que las personas sobrepasen su esperanza de vida, lo cual implica una potencial subestimación de las reservas que se mantienen para cumplir con pasivos futuros, propone un swap de longevidad como alternativa para gestionar el riesgo, utilizando la cobertura natural que surge de posiciones opuestas respecto al mismo riesgo, para aplicarlo a la gestión del riesgo de longevidad que enfrenta el gobierno federal de México. 
Finalmente, López et al. (2020) analizan el rápido crecimiento que ha tenido la población mexicana en su envejecimiento sustituyendo en 1997 su sistema tradicional de reparto por un programa de gestión privada, en el que las contribuciones por o en nombre de los trabajadores activos se depositan en cuentas individuales y se dirigen a un fondo de pensiones de gestión privada. También realizan un análisis exhaustivo para las Sociedades de Inversión Especializadas de Fondos para el Retiro, considerando diferentes niveles de riesgo.

Respecto a las inversiones Kyriakow et al. (2021) muestran avances recientes en el desarrollo de productos de pensiones, los cuales parecen favorecer alternativas al activo libre de riesgo; aplican un suavizador completamente no paramétrico con las covariables y el parámetro suavizado elegido por validación cruzada para pronosticar los rendimientos de las acciones en exceso de diferentes puntos de referencia, incluido el interés a corto plazo, observan que el modelo predice mejores rendimientos futuros anuales para las acciones. Esta es una conclusión crucial para las aplicaciones actuariales que tienen como objetivo proporcionar pronósticos de ingresos reales para los pensionistas.

Por su parte Dopierata y Mosionek (2021) evalúan el impacto de las reformas introducidas en el funcionamiento de los fondos de pensiones abiertos polacos sobre el estilo de gestión, la exposición al riesgo y el rendimiento de las inversiones relacionadas. Examinan si la eliminación del índice de referencia interno para la evaluación de fondos impacta en la eliminación o reducción del comportamiento gregario, los resultados indican que los fondos altamente regulados pueden superar ligeramente los índices de referencia, además expresan que la limitación de las inversiones en instrumentos de deuda del Tesoro resultó claramente un mayor riesgo y volatilidad de los rendimientos. López y Walker (2021) estudian el desempeño de las inversiones en fondos de pensiones chilenos durante el periodo (2003-2017), consideran restricciones regulatorias y de cobertura de divisas, los resultados tienen implicaciones de política para la regulación de inversiones de sistemas de pensiones con características similares al chileno.

Wang et al. (2021) investigan un problema sólido de elección de cartera óptima para un individuo de un plan de pensiones de contribución definida (CD), el individuo tiene un salario estocástico, considera el riesgo de inflación e invierte el patrimonio de su cuenta de pensiones en un mercado financiero que consiste en un activo libre de riesgo, un bono indexado a la inflación y una acción cuya tasa de rendimiento esperada sigue un proceso de reversión a la media, encuentran que la ambigüedad acerca de la 
dinámica del precio de los bonos indexados a la inflación y el precio de las acciones y la tasa de rendimiento esperada tiene diferentes influencias en la estrategia de inversión óptima robusta, y que descuidar la ambigüedad siempre conducirá a la pérdida de utilidad. McCarthy et al. (2021) muestran el rol que tienen los accionistas institucionales en la decisión de la empresa de descargar los pasivos de pensiones a los administradores de riesgos profesionales, encuentran que la probabilidad de transferencias de riesgo de pensión es mayor para las empresas con mayor nivel de propiedad institucional y propietarios institucionales independientes.

La diferencia de este estudio con las investigaciones indicadas en párrafos anteriores consiste en que en este trabajo se propone como alternativa de inversión de los recursos para cada SIEFORE los productos financieros "estructurados"2 o también llamados Notas Estructuradas (NE) Put Spread y Call Spread considerando como subyacentes los precios de cierre de los principales índices sectoriales invertibles (Materiales, Industrial, Servicios Financieros y Consumo Frecuente), como se muestra en la Tabla 1. En el siguiente apartado se enuncia la metodología.

Tabla 1: Principales índices sectoriales invertibles

\begin{tabular}{ll}
\hline Índice & Sector que representa \\
\hline S\&P/BMV Sector Selectivo Materiales & Materiales \\
S\&P/BMV Sector Selectivo Industrial & Industrial \\
S\&P/BMV Sector Selectivo Consumo Frecuente & Consumo Frecuente \\
S\&P/BMV Sector Selectivo Financiero & Servicios Financieros \\
\hline
\end{tabular}

Fuente: elaboración propia con base en la página web de la BMV 2019.

\section{Metodología. Notas Estructuradas Call SpREad y Put Spread}

Con base en el Manual de Metodologías de Valmer³ (2009), se muestra a continuación la metodología para una NE Call Spread.

El precio de la valuación de la NE Call Spread está dada por la siguiente expresión:

\footnotetext{
${ }^{2}$ Los productos financieros estructuradas son un fruto de la ingeniería financiera. La cual busca la innovación de nuevos productos para los inversionistas. Es un hibrido entre un producto derivado y un instrumento financiero de renta fija. Las opciones financieras son el producto derivado más usado para la conformación de los productos financieros "estructurados". Los "estructurados" más comunes son los de capital garantizado, los cuales garantizan al vencimiento al menos no perder la inversión inicial.

${ }^{3}$ Valuación Operativa y Referencias de Mercado S.A. de C.V.
} 


$$
\mathrm{P}_{\mathrm{Vc}}=\mathrm{P}_{\mathrm{B}}+\mathrm{P}_{\mathrm{D}} * \mathrm{~F}
$$

Donde:

F: Factor establecido en el prospecto determinado por el emisor desde el inicio del depósito y ajusta el rendimiento de la NE.

$\mathrm{P}_{\mathrm{Vc}}$ : Precio de valuación de la NE Call Spread.

$\mathrm{P}_{\mathrm{B}}$ : Precio del bono cupón cero.

$\mathrm{P}_{\mathrm{D}}$ : Prima de las opciones implicadas en la estrategia dada por:

$$
\mathrm{P}_{\mathrm{D}}=\mathrm{C}_{\mathrm{K} 1}-\mathrm{C}_{\mathrm{K} 2}
$$

Donde $C_{\mathrm{K} 1}$ y $C_{\mathrm{K} 2}$ son las primas de un Call con precios de ejercicio $\mathrm{K}_{1}$ $\mathrm{y} \mathrm{K}_{2}$, respectivamente.

$$
P_{B}=\frac{V N}{\left(1+T L R \frac{n}{360}\right)}
$$

Donde:

VN: Valor nominal de la NE Call Spread.

$\mathrm{n}$ : Número de días por vencer de la NE.

TLR: Tasa de interés libre de riesgo (CETES) asociada al número de días por vencer.

El valor de la prima de ambas opciones se obtiene mediante la fórmula general de Black y Scholes (1973) y Merton (1973), puesto que ambas opciones se ejercen únicamente al vencimiento de la NE.

$$
C_{K}=S_{0} N\left(d_{1}\right)-K e^{-T L R * T} N\left(d_{2}\right)
$$

Donde:

$d_{1}, d_{2}$ y $T$ se definen como:

$$
d_{1}=\frac{\ln \left(\frac{S_{0}}{K}\right)+\left(T L R+\frac{\sigma^{2}}{2}\right) T}{\sigma \sqrt{T}}, d_{2}=d_{1}-\sigma \sqrt{T} \text { y } T=\frac{n}{365}
$$

Donde:

$\mathrm{C}_{\mathrm{k}}$ : Prima de la opción tipo Call con precio de ejercicio .

$\mathrm{S}_{0}$ : Valor inicial del subyacente.

$\mathrm{N}(\bullet)$ : Probabilidad acumulada de la distribución normal estándar en el valor $(\bullet)$. 
$\sigma$ : Volatilidad del rendimiento del subyacente.

Por otro lado, el precio de la valuación de la NE Put Spread está dada por la siguiente expresión:

$\mathrm{P}_{\mathrm{Vp}}=\mathrm{P}_{\mathrm{B}}+\mathrm{P}_{\mathrm{D}} * \mathrm{~F}$

Donde:

F: Factor establecido en el prospecto determinado por el emisor desde el inicio del depósito y ajusta el rendimiento de la NE.

$\mathrm{P}_{\mathrm{vp}}:$ Precio de valuación de la NE Put Spread.

$\mathrm{P}_{\mathrm{B}}^{\mathrm{v}}$ : Precio del bono cupón cero.

$\mathrm{P}_{\mathrm{D}}$ : Prima de las opciones implicadas en la estrategia dada por:

$$
\mathrm{P}_{\mathrm{D}}=\mathrm{C}_{\mathrm{K} 2}-\mathrm{C}_{\mathrm{K} 1}
$$

Donde $P_{\mathrm{K} 1}$ y $P_{\mathrm{K} 2}$ son las primas de un $P u t$ con precios de ejercicio $K_{1}$ y $K_{2}$, respectivamente.

La valuación tanto del bono cupón cero y de los puts incorporados en la $\mathrm{NE}$ se determinan de la siguiente manera:

$$
P_{B}=\frac{V N}{\left(1+T L R \frac{n}{360}\right)}
$$

Donde:

VN: Valor nominal de la NE Put Spread. n: Número de días por vencer de la NE.

TLR: Tasa de interés libre de riesgo (CETES) asociada al número de días por vencer.

$$
P_{K}=K e^{-T L R * T} N\left(-d_{2}\right)-S_{0} N\left(-d_{1}\right)
$$

Donde:

$d_{1}, d_{2}$ y $T$ se definen como:

$$
\begin{gathered}
d_{1}=\frac{\ln \left(\frac{S_{0}}{K}\right)+\left(T L R+\frac{\sigma^{2}}{2}\right) T}{\sigma \sqrt{T}}, d_{2}=d_{1}-\sigma \sqrt{T}, T=\frac{n}{365}, N\left(-d_{2}\right) \\
=1-N\left(d_{2}\right) y N\left(-d_{1}\right)=1-N\left(d_{1}\right)
\end{gathered}
$$


Donde:

$\mathrm{P}_{\mathrm{k}}$ : Prima de la opción tipo Put con precio de ejercicio $K$.

$\mathrm{S}_{0}$ : Valor inicial del subyacente.

$\mathrm{N}(\bullet)$ : Probabilidad acumulada de la distribución normal estándar en el valor $(\bullet)$.

$\sigma$ : Volatilidad del rendimiento del subyacente.

\section{Análisis de Resultados}

De la página de la CONSAR se extrajo la información del último trimestre del año 2018 y de los primeros dos trimestres del año 2019, para realizar un comparativo al cierre de marzo y junio de 2019 entre la información real reflejada por dicha institución donde muestra resultados para cada tipo de SIEFORE al diversificar los recursos de los trabajadores contra la alternativa de invertir la totalidad de los recursos en el producto financiero estructurado.

La información reportada por la CONSAR en su último trimestre del año 2018 se muestra en la Tabla 2.

Los resultados obtenidos para el Sector Materiales al trimestre siguiente para cada tipo de SIEFORE se muestra en la Tabla 3.

La Tabla 3 muestra que en todos los tipos de SIEFORES se obtuvieron ganancias con la NE Put Spread; obteniendo así 3,791,132,074,078 pesos mexicanos como valor de la cartera total a finales del mes de marzo, dicha cantidad representa una ganancia total en el trimestre de 14.43 por ciento.

Los resultados obtenidos para el Sector Industrial al cierre del primer trimestre del 2019 para cada tipo de SIEFORE se muestra en la Tabla 4:.La Tabla 4 muestra que en todos los tipos de SIEFORES se obtuvieron ganancias con la NE Call Spread; obteniendo así 3,404,127,795,731 pesos mexicanos como valor de la cartera total a finales del mes de marzo, dicha cantidad representa una ganancia total en el trimestre de 2.75 por ciento.

Los resultados obtenidos para el Sector Financiero al cierre del primer trimestre del 2019 para cada tipo de SIEFORE se muestra en la Tabla 5.

La Tabla 5 muestra que en todos los tipos de SIEFORES se obtuvieron ganancias con la NE Call Spread; obteniendo así 3,510,953,926,106 pesos mexicanos como valor de la cartera total a finales del mes de marzo, dicha cantidad representa una ganancia total en el trimestre de 5.97 por ciento.

Los resultados obtenidos en el Sector Consumo Frecuente al cierre del primer trimestre del 2019 para cada tipo de SIEFORE se muestra en la Tabla 6. 
Marco jurídico de las pensiones mexicanas: análisis empírico mediante productos ... / H.A. OLIVARES AGUAYO et al.

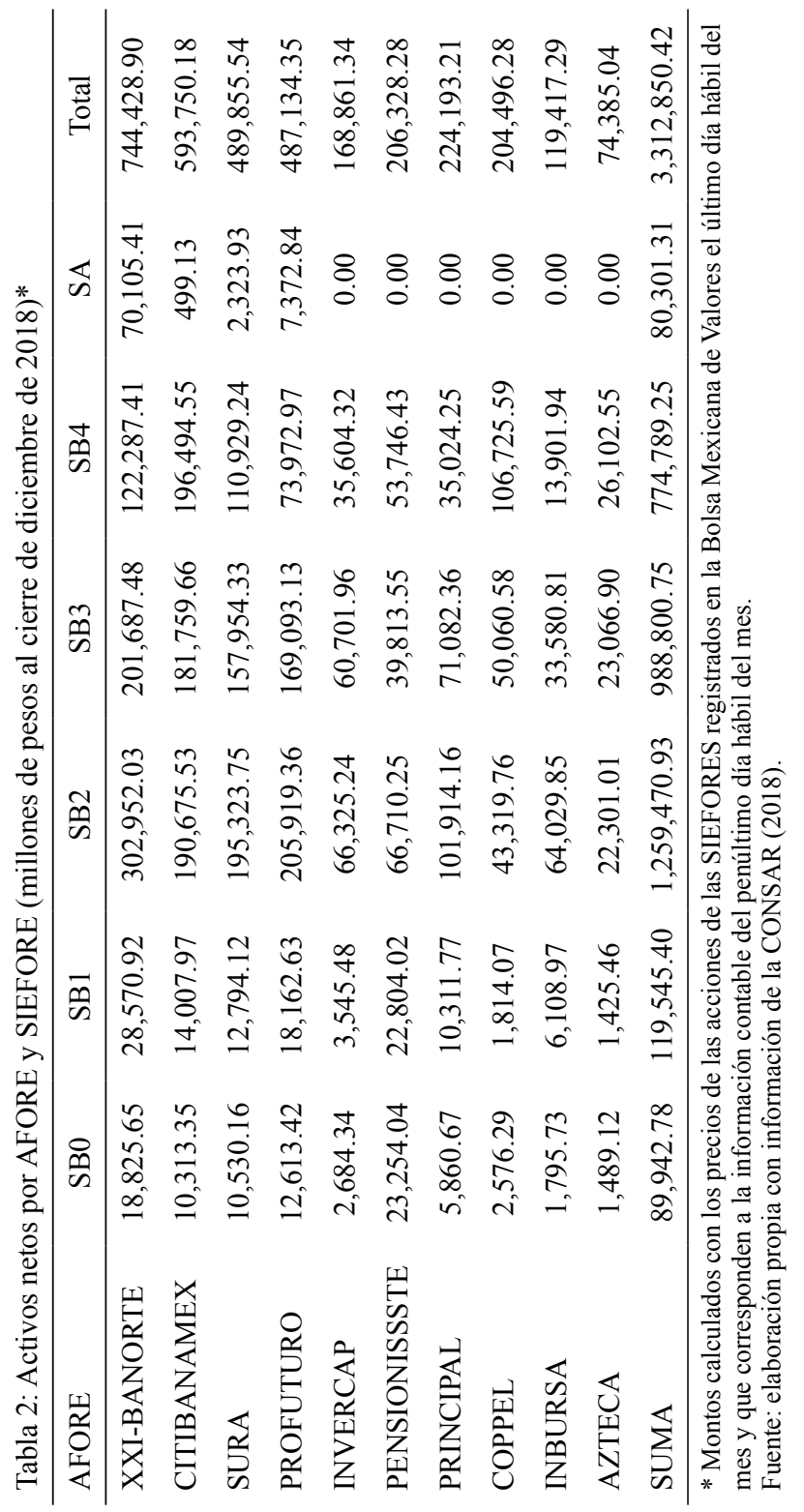




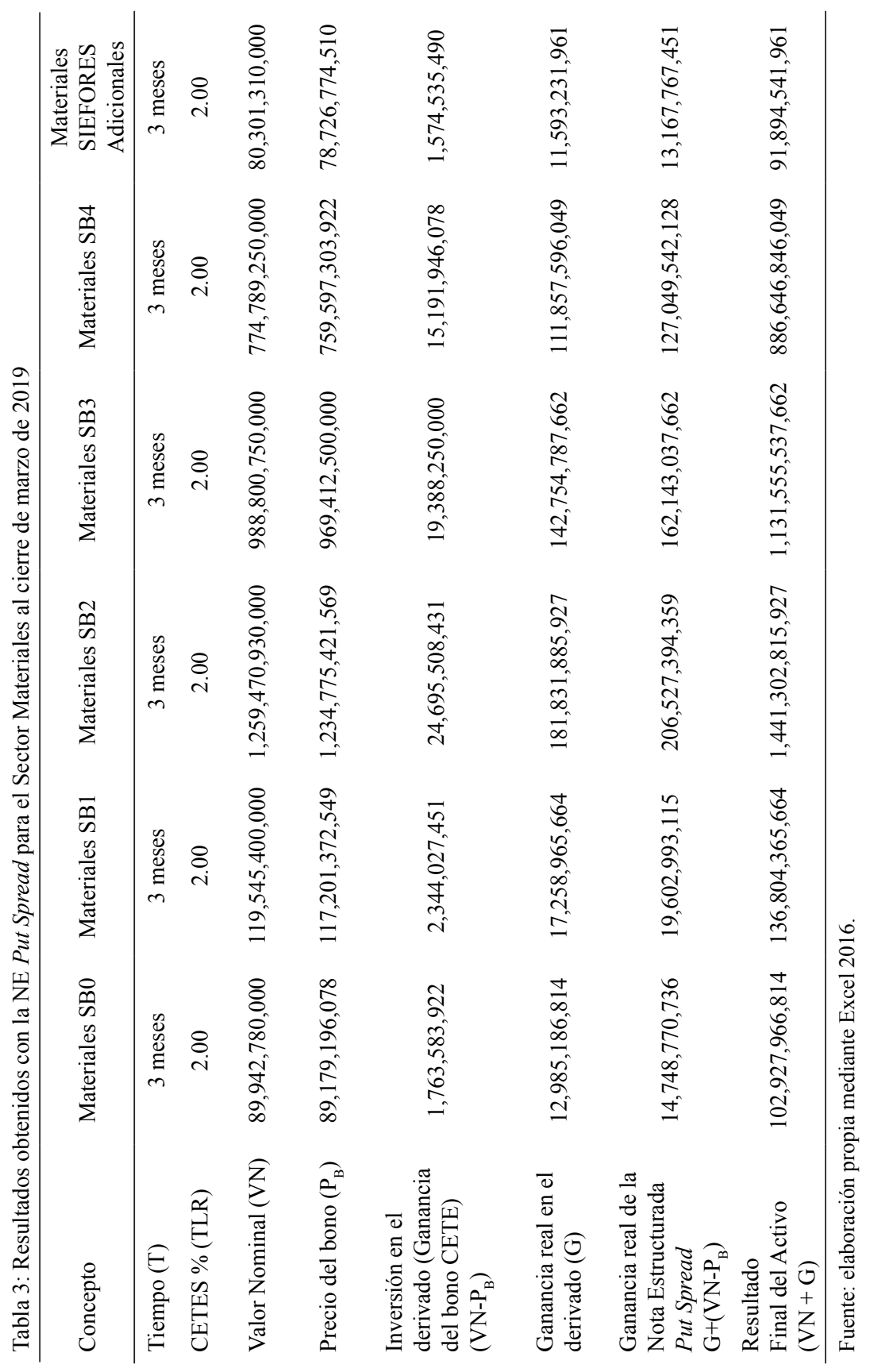


Marco jurídico de las pensiones mexicanas: análisis empírico mediante productos ... / H.A. OLIVARES AGUAYO et al.

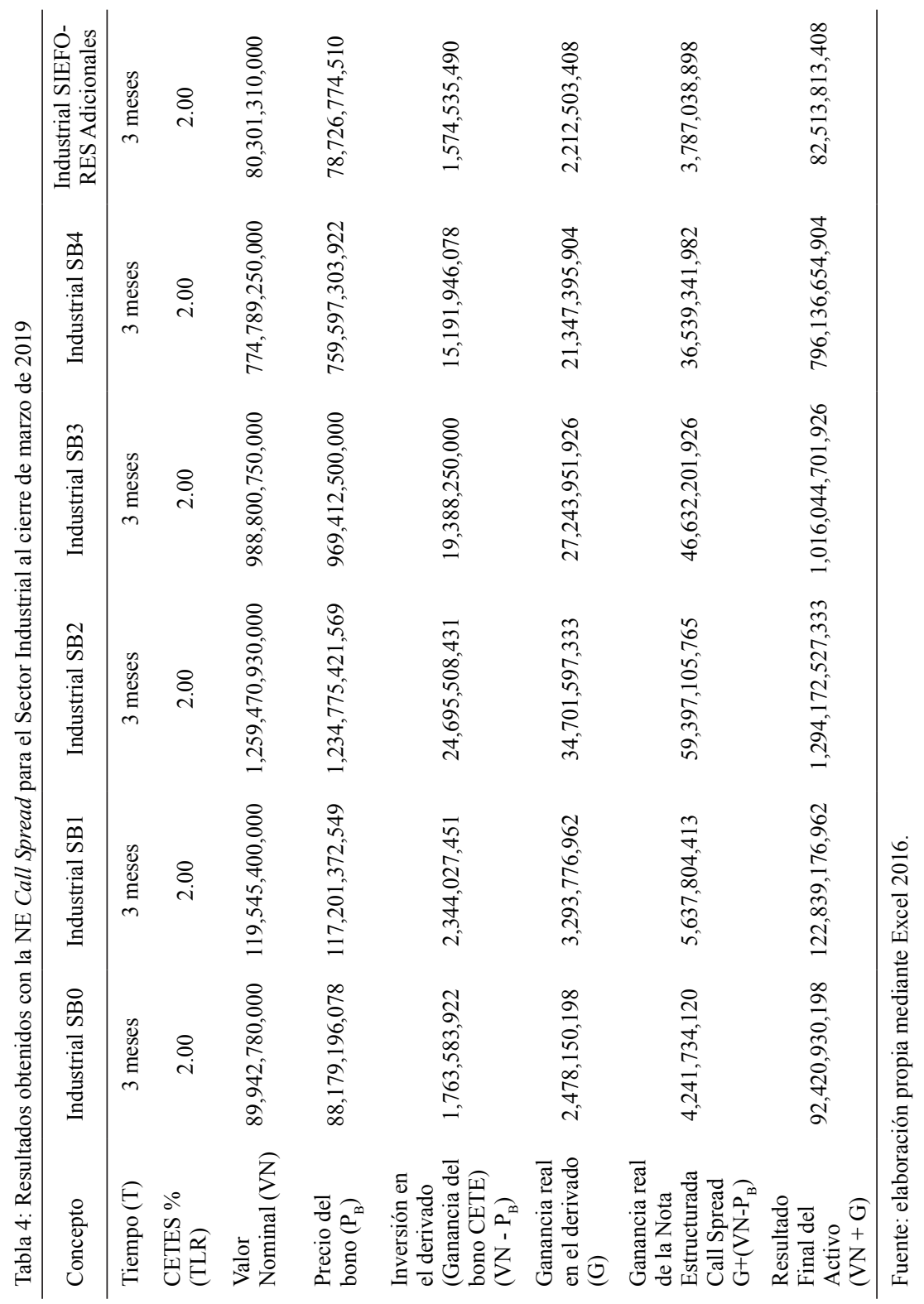




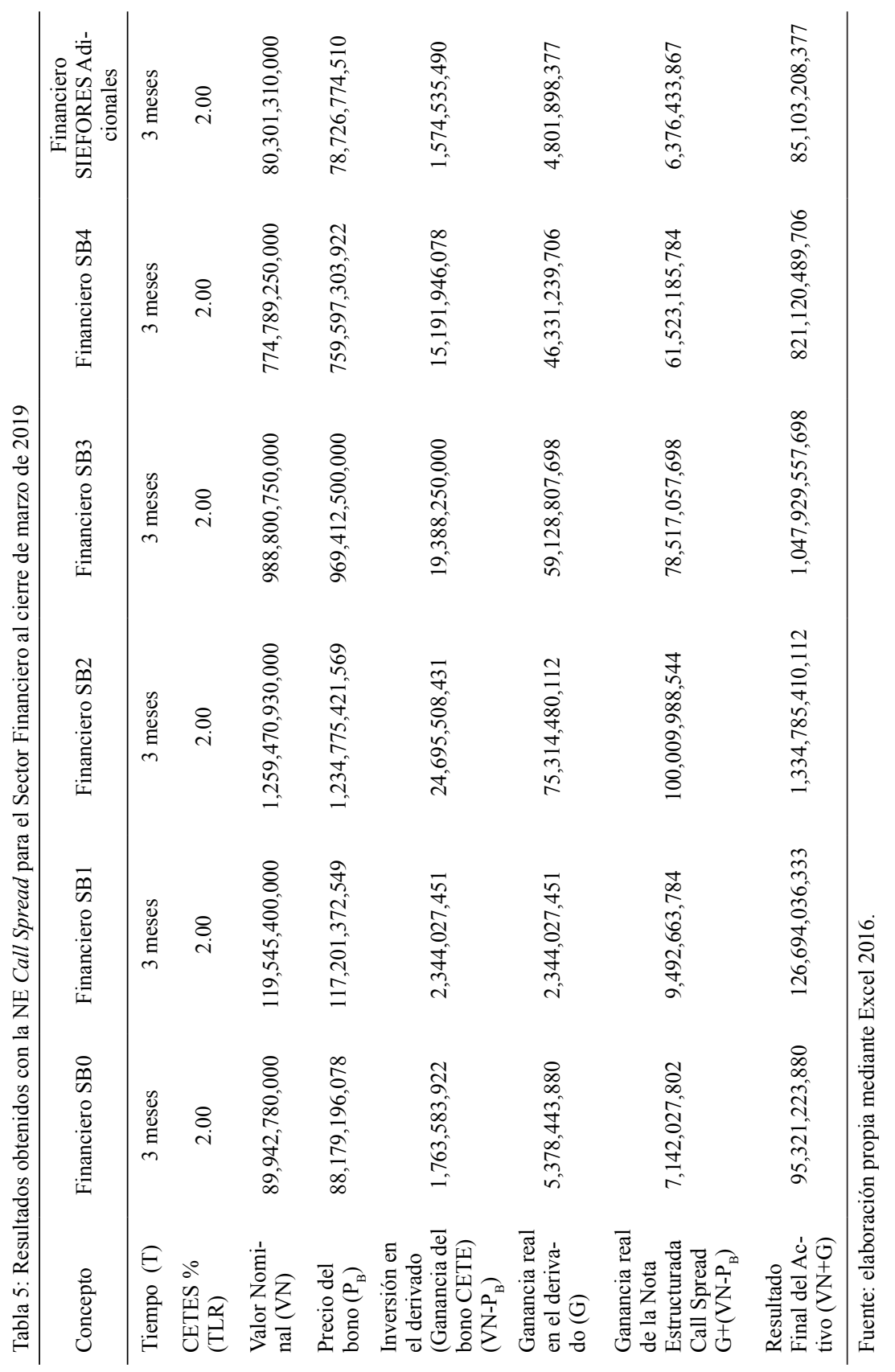


La Tabla 6 muestra que en todos los tipos de SIEFORES se obtuvieron ganancias con la NE Put Spread; obteniendo así 3,462,106,545,935 pesos mexicanos como valor de la cartera total a finales del mes de marzo, dicha cantidad representa una ganancia total en el trimestre de 4.50 por ciento.

Al comparar los resultados obtenidos contra la información real reflejada por la CONSAR que se muestra en la Tabla 7.

La Tabla 7 muestra un valor total de la cartera por 3,537,067,450,000 pesos mexicanos a finales del mes de marzo, dicha cantidad representa una ganancia total en el trimestre de 6.76 por ciento. Sin embargo, es importante decir que no en todos los tipos de SIEFORES se obtuvieron ganancias con las inversiones realizadas. Pues, la SB0 y SB1 donde se encuentran los recursos de las personas de 60 años o más se observan pérdidas reales de 0.14 por ciento y 0.28 por ciento respectivamente al cierre de marzo del presente año.

La Tabla 8 muestra el porcentaje de inversión en instrumentos financieros, incluyendo los "estructurados".

En la Tabla 8 se muestran los porcentajes de inversión realizadas en "estructurados" para los diferentes tipos de SIEFORES. El rango de inversión va desde cero para la SB0 hasta 7.3 por ciento en la SB3. En general estos porcentajes son pequeños. Sin embargo, de dicha Tabla, hay que destacar dos cosas: la primera observación es que en las tres SIEFORES que tienen un mayor porcentaje de inversión (SB2, SB3 y SB4) son las SIEFORES que tuvieron una mayor cantidad de ganancia real según la información reportada por la CONSAR al cierre del mes de marzo del presente año. Dicha ganancia porcentual se encuentra en un rango de 5.92 a 9.98 por ciento en todos los casos respecto al trimestre anterior, y en términos monetarios para la SB2 equivale a 74,683,830,000 pesos mexicanos, para la SB3 de 65,396,330,000 pesos mexicanos y para la SB4 77,358,751,000 pesos mexicanos; en los tres casos la ganancia es más menos de setenta mil millones de pesos mexicanos. Para la SB0 y la SB1 se observan pérdidas con únicamente de 0 y 0.9 por ciento de inversión en "estructurados".

A continuación, se muestra en la Tabla 9 un resumen comparativo de las diferencias monetarias respecto a los resultados obtenidos y los valores reales mostrados por la CONSAR para cada SIEFORE en los cierres del último trimestre de 2018 y el primero de 2019. 


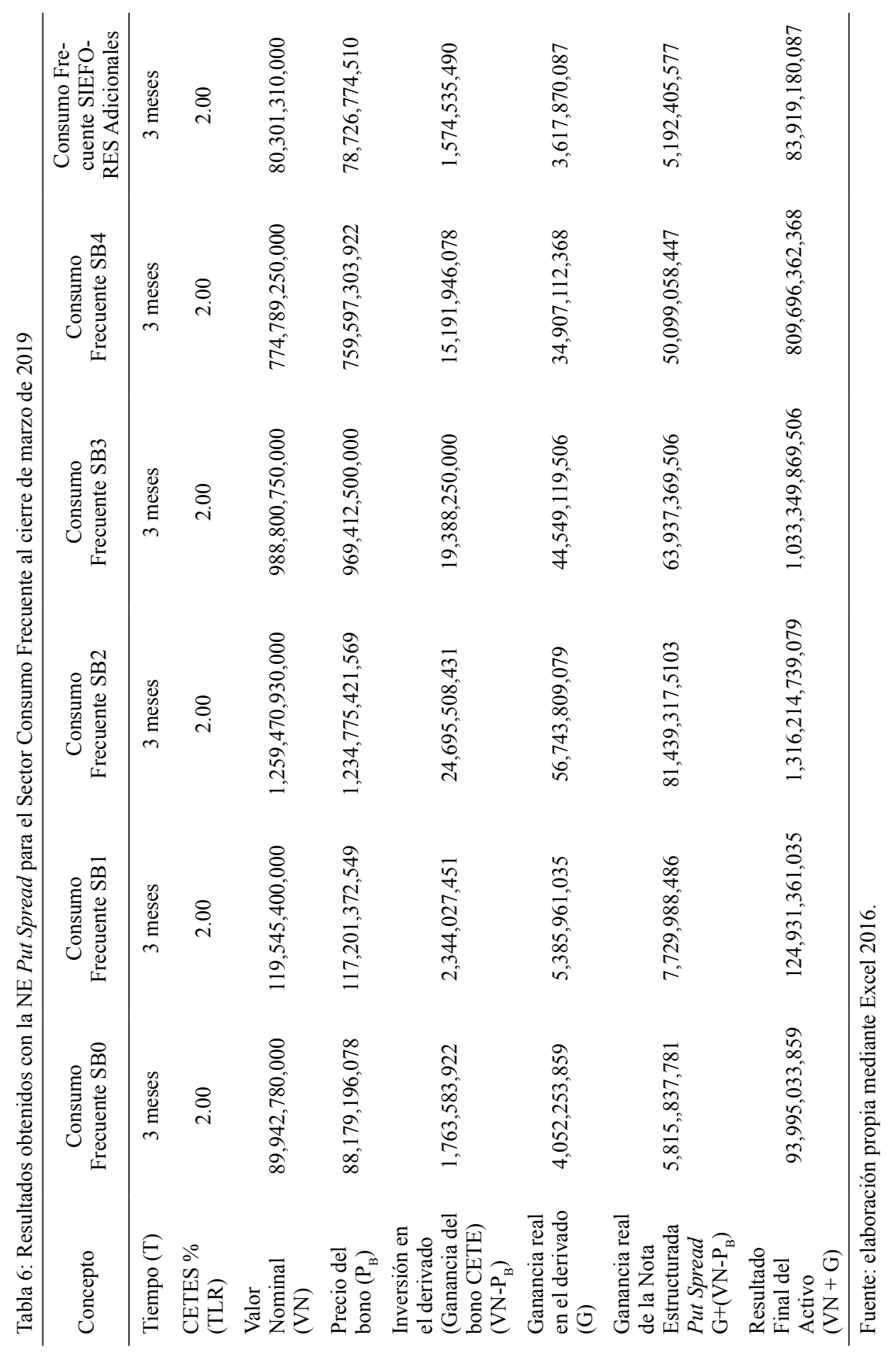


Marco jurídico de las pensiones mexicanas: análisis empírico mediante productos ... / H.A. OLIVARES AGUAYO et al.

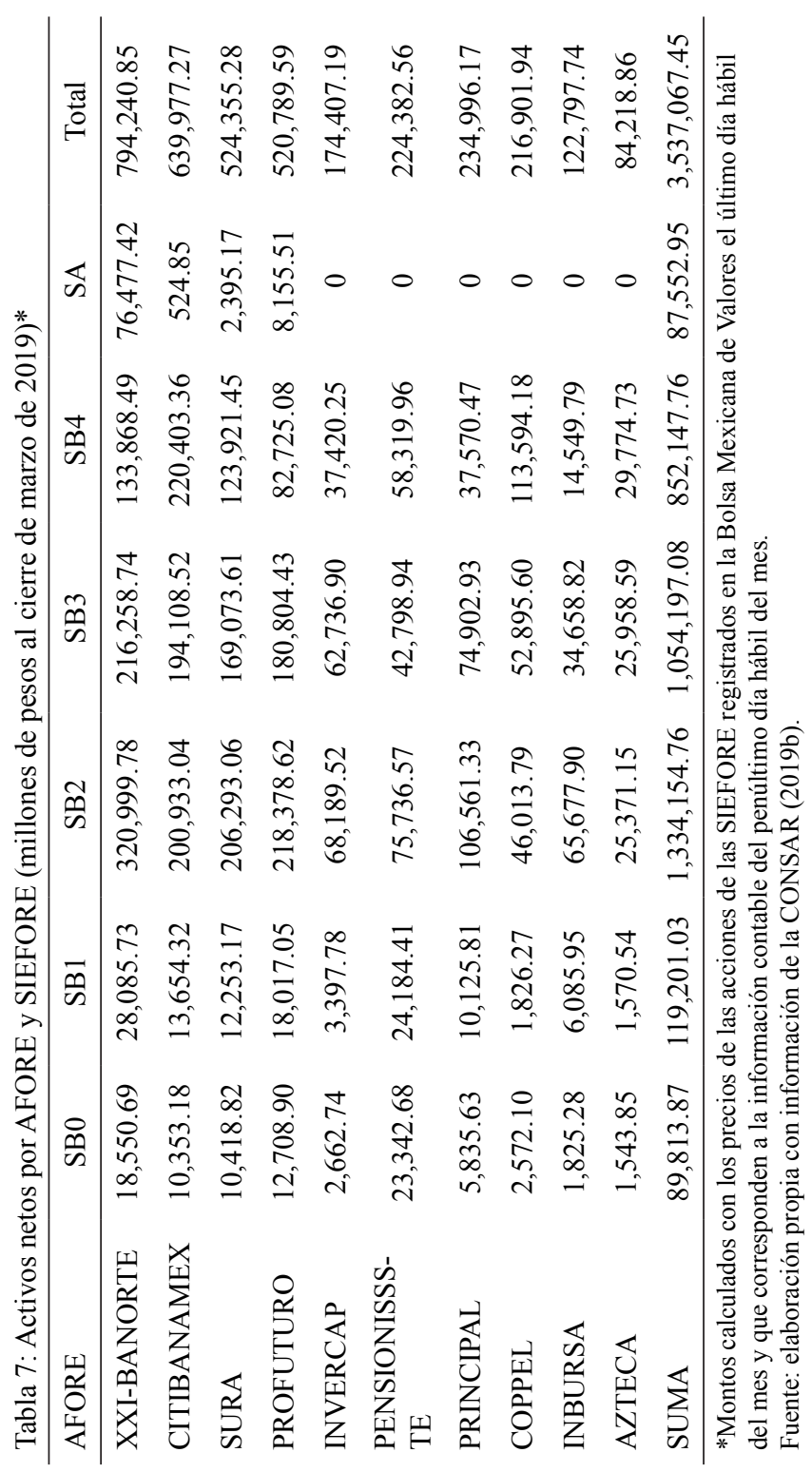


Tabla 8: Composición de las carteras de inversión de las SIEFORES (porcentaje respecto al valor de las carteras administradas)

\begin{tabular}{|c|c|c|c|c|c|c|c|}
\hline $\begin{array}{l}\text { Tipo de } \\
\text { instrumento }\end{array}$ & $\begin{array}{c}\text { SB0 } \\
\%\end{array}$ & $\begin{array}{c}\text { SB1 } \\
\%\end{array}$ & $\begin{array}{c}\text { SB2 } \\
\%\end{array}$ & $\begin{array}{c}\text { SB3 } \\
\%\end{array}$ & $\begin{array}{c}\text { SB4 } \\
\%\end{array}$ & $\begin{array}{l}\text { SA } \\
\%\end{array}$ & $\begin{array}{c}\text { Total } \\
\%\end{array}$ \\
\hline $\begin{array}{l}\text { Pesos Deuda } \\
\text { Gubernamental }\end{array}$ & 100.00 & 65.90 & 51.30 & 47.90 & 43.40 & 69.40 & 50.60 \\
\hline $\begin{array}{l}\text { Divisas Deuda } \\
\text { Gubernamental }\end{array}$ & 0.00 & 0.70 & 0.70 & 0.90 & 0.70 & 0.10 & 0.70 \\
\hline Aerolíneas & 0.00 & 0.10 & 0.00 & 0.00 & 0.10 & 0.00 & 0.00 \\
\hline Alimentos & 0.00 & 0.50 & 0.80 & 0.90 & 0.70 & 0.00 & 0.80 \\
\hline Automotriz & 0.00 & 0.10 & 0.20 & 0.20 & 0.30 & 0.10 & 0.20 \\
\hline $\begin{array}{l}\text { Banca de } \\
\text { Desarrollo }\end{array}$ & 0.00 & 1.80 & 1.50 & 1.30 & 1.00 & 1.10 & 1.30 \\
\hline Bancario & 0.00 & 1.20 & 1.20 & 1.20 & 1.00 & 0.80 & 1.10 \\
\hline Bebidas & 0.00 & 0.70 & 0.60 & 0.60 & 0.60 & 0.20 & 0.60 \\
\hline Cemento & 0.00 & 0.00 & 0.00 & 0.00 & 0.00 & 0.00 & 0.00 \\
\hline $\begin{array}{l}\text { Centros } \\
\text { Comerciales }\end{array}$ & 0.00 & 0.10 & 0.10 & 0.10 & 0.10 & 0.00 & 0.10 \\
\hline Consumo & 0.00 & 0.30 & 0.30 & 0.30 & 0.40 & 0.10 & 0.30 \\
\hline $\begin{array}{l}\text { Empresas produc- } \\
\text { tivas del estado }\end{array}$ & 0.00 & 4.10 & 2.90 & 2.60 & 2.40 & 2.10 & 2.60 \\
\hline Estados & 0.00 & 0.80 & 0.40 & 0.40 & 0.30 & 0.60 & 0.40 \\
\hline Europesos & 0.00 & 2.90 & 3.10 & 3.00 & 2.80 & 0.70 & 2.90 \\
\hline $\begin{array}{l}\text { Grupos Indus- } \\
\text { triales }\end{array}$ & 0.00 & 0.80 & 0.60 & 0.60 & 0.50 & 0.00 & 0.60 \\
\hline Infraestructura & 0.00 & 5.00 & 3.90 & 3.80 & 4.30 & 1.40 & 3.80 \\
\hline Inmobiliario & 0.00 & 0.00 & 0.00 & 0.10 & 0.10 & 0.00 & 0.10 \\
\hline Otros & 0.00 & 1.10 & 1.00 & 0.90 & 0.90 & 0.30 & 0.90 \\
\hline Papel & 0.00 & 0.10 & 0.10 & 0.10 & 0.10 & 0.00 & 0.10 \\
\hline $\begin{array}{l}\text { Servicios } \\
\text { Financieros }\end{array}$ & 0.00 & 0.40 & 0.40 & 0.50 & 0.50 & 0.10 & 0.40 \\
\hline Siderúrgica & 0.00 & 0.00 & 0.00 & 0.00 & 0.00 & 0.00 & 0.00 \\
\hline Telecom & 0.00 & 1.10 & 1.30 & 1.10 & 0.90 & 0.20 & 1.10 \\
\hline Transporte & 0.00 & 0.40 & 0.50 & 0.60 & 0.60 & 0.00 & 0.50 \\
\hline Vivienda & 0.00 & 2.30 & 1.50 & 1.40 & 1.30 & 1.10 & 1.40 \\
\hline Estructurados & 0.00 & 0.90 & 6.00 & 7.30 & 6.60 & 0.10 & 6.10 \\
\hline FIBRAS & 0.00 & 1.50 & 2.80 & 2.70 & 2.80 & 2.10 & 2.60 \\
\hline Renta Variable & 0.00 & 2.00 & 5.40 & 6.30 & 8.90 & 4.70 & 6.20 \\
\hline $\begin{array}{l}\text { Deuda } \\
\text { Internacional }\end{array}$ & 0.00 & 1.20 & 1.00 & 1.00 & 1.10 & 0.00 & 1.00 \\
\hline $\begin{array}{l}\text { Renta Variable } \\
\text { Internacional }\end{array}$ & 0.00 & 3.90 & 11.90 & 14.00 & 17.20 & 14.30 & 13.20 \\
\hline Mercancías & 0.00 & 0.00 & 0.30 & 0.40 & 0.40 & 0.60 & 0.30 \\
\hline Total & 100.00 & 100.00 & 100.00 & 100.00 & 100.00 & 100.00 & 100.00 \\
\hline
\end{tabular}

Cifras al cierre de diciembre 2018.

Fuente: CONSAR (2018). 
Marco jurídico de las pensiones mexicanas: análisis empiríco mediante productos ... / H.A. OLIVARES AGUAYO et al.

Tabla 9: Diferencias monetarias en el último trimestre de 2018 y el primer trimestre de 2019 en SIEFORES (cifras en millones de pesos)

\begin{tabular}{lccccccc}
\hline Concepto & SB0 & SB1 & SB2 & SB3 & SB4 & SA & Total \\
\hline $\begin{array}{l}\text { Diferencias } \\
\text { reales }\end{array}$ & -128.91 & -344.37 & $74,683.83$ & $65,396.33$ & $77,358.51$ & $7,251.64$ & $224,217.03$ \\
$\begin{array}{l}\text { Diferencias } \\
\text { Materiales }\end{array}$ & $12,985.20$ & $17,258.97$ & $181,831.89$ & $142,754.79$ & $111,857.60$ & $11,593.23$ & $478,281.65$ \\
$\begin{array}{l}\text { Diferencias } \\
\text { Industrial }\end{array}$ & $2,478.15$ & $3,293.78$ & $34,701.60$ & $27,243.95$ & $21,347.40$ & $2,212.50$ & $91,277.38$ \\
$\begin{array}{l}\text { Diferencias } \\
\text { Financiero }\end{array}$ & $5,378.44$ & $7,148.64$ & $75,314.48$ & $59,128.81$ & $46,331.24$ & $4,891.90$ & $198,103.51$ \\
$\begin{array}{l}\text { Diferencias } \\
\text { Consumo }\end{array}$ & $4,052.25$ & $5,385.96$ & $56,743.81$ & $44,549.12$ & $34,907.11$ & 3617.87 & $149,256.13$ \\
Frecuente & & & & & & & \\
\hline
\end{tabular}

Fuente: elaboración propia mediante Excel 2016.

En la Tabla 9 se puede apreciar que para todos los tipos de SIEFORES la inversión en el Sector Materiales fue la que mostró mejores resultados. Pues, las ganancias respecto al trimestre anterior son las más elevadas, lo anterior se resume en una ganancia total de 478,281.65 millones de pesos mexicanos, que es más del doble que lo mostrado por la CONSAR mediante la diversificación de activos. Asimismo, en los casos mostrados de los otros sectores, se observa que para el caso de la SB0 y SB1 siempre se obtuvieron mayores ganancias a las mostradas por la CONSAR, mejor dicho, la CONSAR refleja pérdidas para dichas SIEFORES de 128.91 y 344.37 millones de pesos, respectivamente.

Ampliando el análisis de esta investigación con información de los primeros dos trimestres de 2019, y partiendo de la información mostrada por la CONSAR correspondiente a la Tabla 7.

Se analizan los resultados obtenidos para el Sector Materiales al trimestre siguiente para cada tipo de SIEFORE como se muestra en la Tabla 10:

La Tabla 10 muestra que en todos los tipos de SIEFORES se obtuvieron ganancias con la NE Put Spread; obteniendo así 3,847,398,072,787 pesos mexicanos como valor de la cartera total a finales del mes de junio, dicha cantidad representa una ganancia total en el trimestre de 8.77 por ciento.

Los resultados obtenidos para el Sector Industrial al trimestre siguiente para cada tipo de SIEFORE se muestra en la Tabla 11.

La Tabla 11 muestra que en todos los tipos de SIEFORES se obtuvieron ganancias con la NE Call Spread; obteniendo así 3,567,090,431,798 pesos mexicanos como valor de la cartera total a finales del mes de junio, dicha cantidad representa una ganancia total en el trimestre de 0.84 por ciento. 


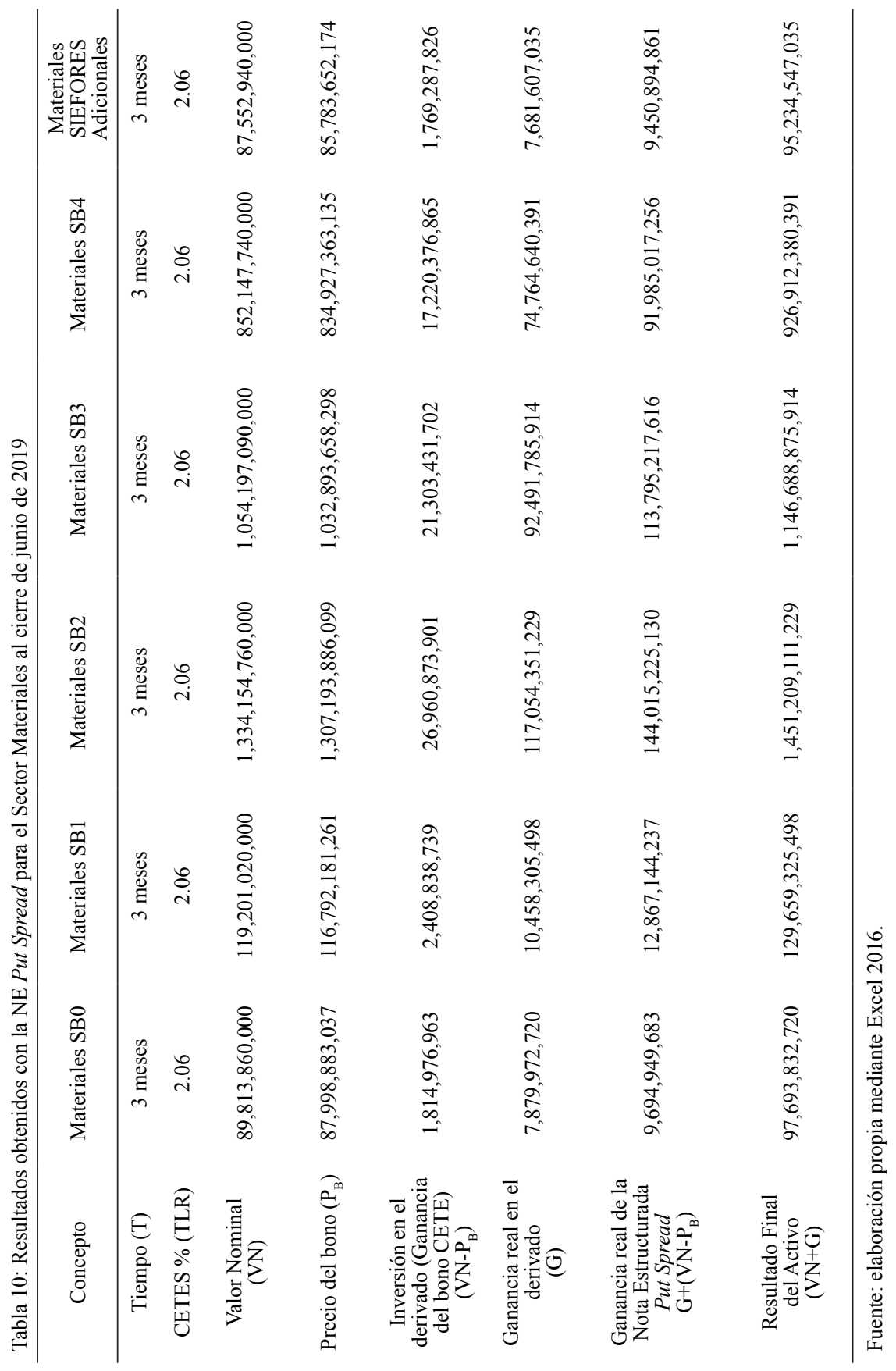


Marco jurídico de las pensiones mexicanas: análisis empírico mediante productos ... / H.A. OLIVARES AGUAYO et al.

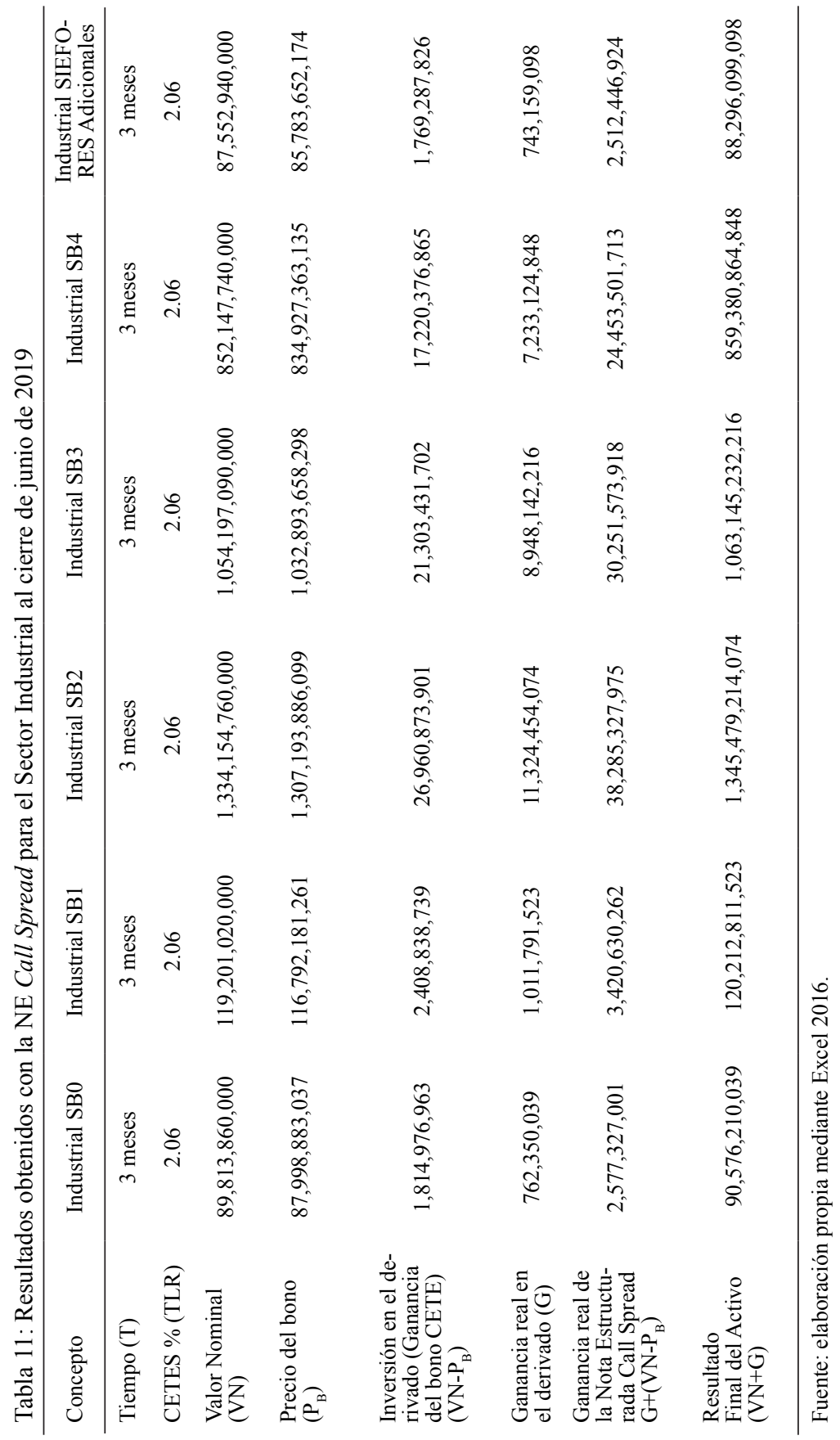


Los resultados obtenidos para el Sector Financiero al trimestre siguiente para cada tipo de SIEFORE se muestra en la Tabla 12.

La Tabla 12 muestra que en todos los tipos de SIEFORES se obtuvieron ganancias con la NE Call Spread; obteniendo así 3,602,419,611,985 pesos mexicanos como valor de la cartera total a finales del mes de junio, dicha cantidad representa una ganancia total en el trimestre de 1.84 por ciento.

Los resultados obtenidos en el Sector Consumo Frecuente al trimestre siguiente para cada tipo de SIEFORE se muestra en la Tabla 13.

La Tabla 13 muestra que en todos los tipos de SIEFORES se obtuvieron ganancias con la NE Put Spread; obteniendo así 3,571,474,498,598 pesos mexicanos como valor de la cartera total a finales del mes de junio, dicha cantidad representa una ganancia total en el trimestre de 0.97 por ciento.

Al comparar los resultados obtenidos contra la información real reflejada por la CONSAR que se muestra en la Tabla 14.

La Tabla 14 muestra un valor total de la cartera por 3,692,740,700,000 pesos mexicanos a finales del mes de junio, dicha cantidad representa una ganancia total en el trimestre de 4.4 por ciento. Sin embargo, es importante enfatizar que no en todos los tipos de SIEFORES se obtuvieron ganancias con las decisiones de inversión de los recursos realizadas. Ya que, para la SB1 donde se encuentran los recursos de las personas de 60 años o más se tuvo pérdidas de 0.73 por ciento al cierre de junio del presente año.

A continuación, en la Tabla 15, se muestra la diversificación en instrumentos financieros de la CONSAR al cierre de marzo de 2019.

En la Tabla 15 se muestran los porcentajes de inversión realizadas en "estructurados" para los diferentes tipos de SIEFORES. El rango de inversión va desde cero para la SB0 hasta 7.3 por ciento en la SB3. Los resultados son consistentes con los mostrados en el trimestre anterior. La ganancia para las SIEFORES SB2, SB3, y SB4 oscila en un rango de 3.56 a 6.38 por ciento en todos los casos respecto al cierre del trimestre anterior, y en términos monetarios para la SB2 equivale a 47,524,810,000 pesos mexicanos, para la SB3 de 49, 223, 800,000 pesos mexicanos y para la SB4 54,376,770,000 pesos mexicanos; en los tres casos prácticamente la ganancia es de cincuenta mil millones de pesos mexicanos. Por otro lado, se observa que en la SB1 donde se invirtió únicamente 0.9 por ciento en "estructurados". Fue la única SIEFORE que tuvo pérdidas reales en el análisis realizado de dicho trimestre.

En resumen, a continuación, se muestra en la Tabla 16 un comparativo de las diferencias monetarias respecto a los resultados obtenidos y los valores reales mostrados por la CONSAR para cada SIEFORE en los cierres del primer y segundo trimestre del presente año. 
Marco jurídico de las pensiones mexicanas: análisis empírico mediante productos ... / H.A. OLIVARES AGUAYO et al.

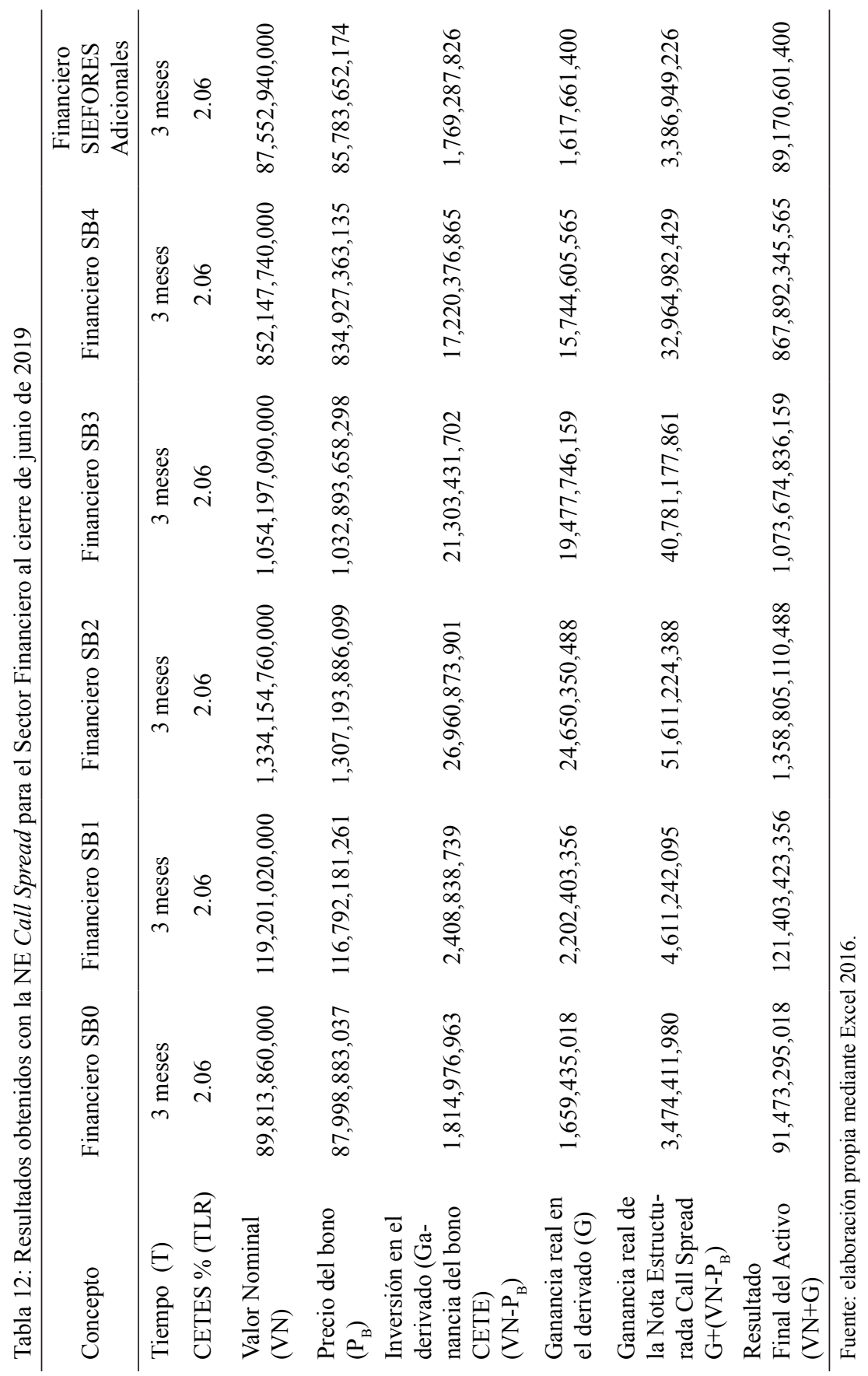




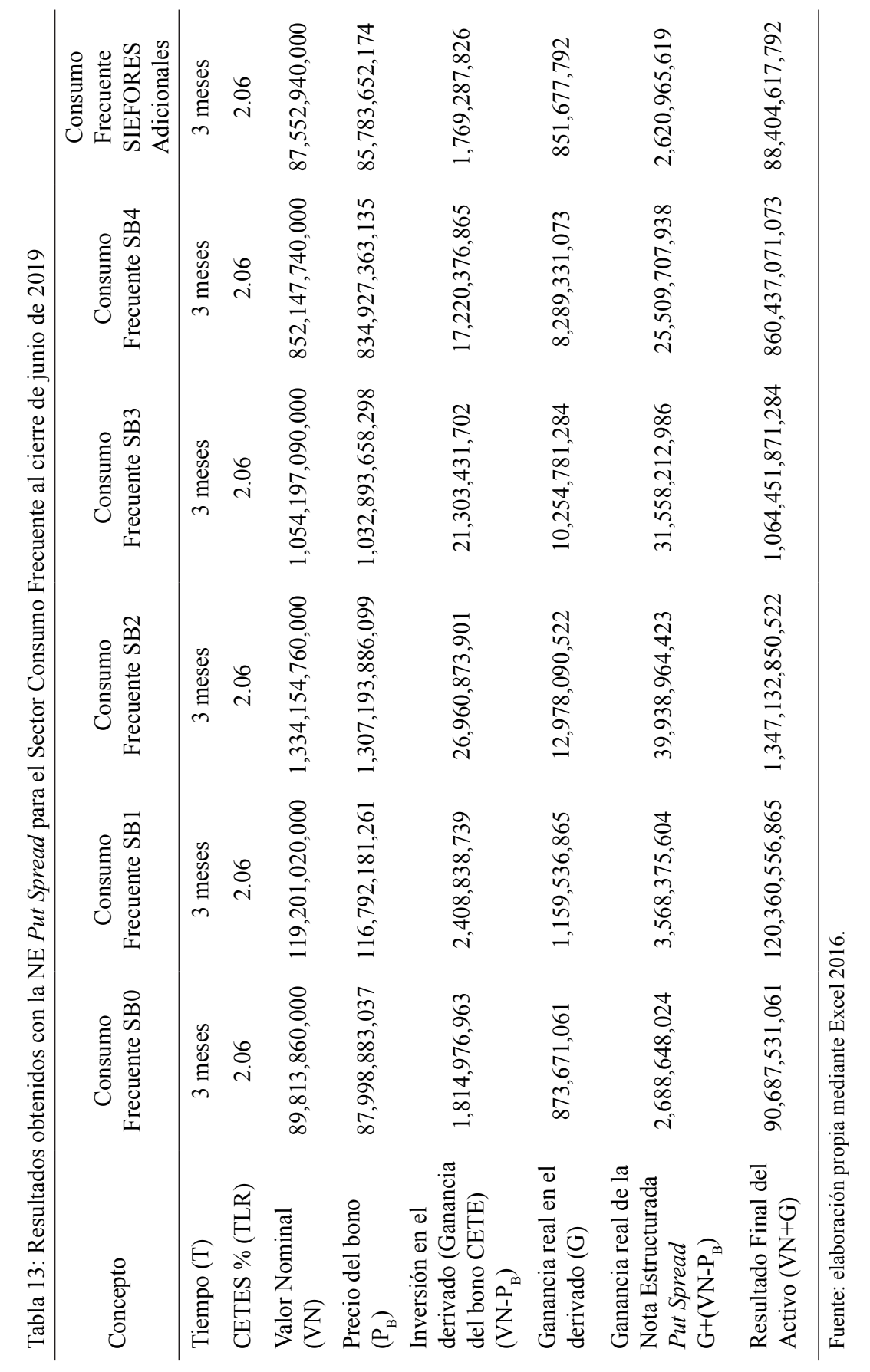


Marco jurídico de las pensiones mexicanas: análisis empírico mediante productos ... / H.A. OLIVARES AGUAYO et al.

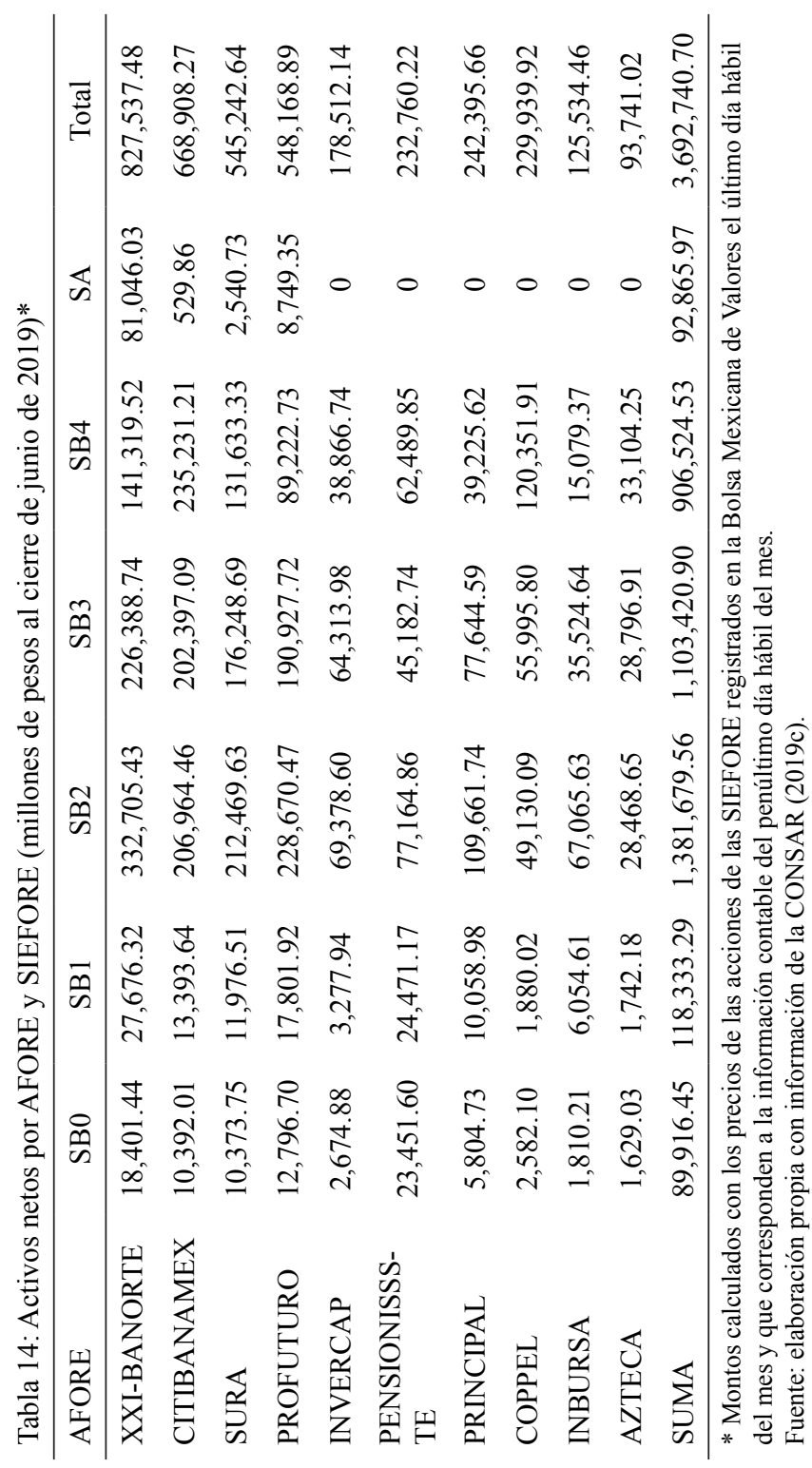


Tabla 15: Composición de las carteras de inversión de las SIEFORES (porcentaje respecto al valor de las carteras administradas)

\begin{tabular}{|c|c|c|c|c|c|c|c|}
\hline $\begin{array}{l}\text { Tipo de } \\
\text { instrumento }\end{array}$ & $\begin{array}{c}\text { SB0 } \\
\%\end{array}$ & $\begin{array}{c}\mathrm{SB} 1 \\
\%\end{array}$ & $\begin{array}{c}\text { SB2 } \\
\%\end{array}$ & $\begin{array}{c}\text { SB3 } \\
\%\end{array}$ & $\begin{array}{c}\text { SB4 } \\
\%\end{array}$ & $\begin{array}{l}\text { SA } \\
\%\end{array}$ & $\begin{array}{c}\text { Total } \\
\%\end{array}$ \\
\hline $\begin{array}{l}\text { Pesos Deuda } \\
\text { Gubernamental }\end{array}$ & 100.0 & 64.30 & 53.60 & 51.20 & 46.80 & 72.60 & 53.10 \\
\hline $\begin{array}{l}\text { Divisas Deuda } \\
\text { Gubernamental }\end{array}$ & 0.00 & 0.70 & 0.60 & 0.90 & 0.80 & 0.10 & 0.70 \\
\hline Aerolíneas & 0.00 & 0.10 & 0.00 & 0.00 & 0.10 & 0.00 & 0.00 \\
\hline Alimentos & 0.00 & 0.60 & 0.90 & 0.90 & 0.70 & 0.00 & 0.80 \\
\hline Automotriz & 0.00 & 0.10 & 0.20 & 0.20 & 0.30 & 0.10 & 0.20 \\
\hline $\begin{array}{l}\text { Banca de } \\
\text { Desarrollo }\end{array}$ & 0.00 & 1.80 & 1.40 & 1.10 & 0.90 & 0.90 & 1.10 \\
\hline Bancario & 0.00 & 1.80 & 1.30 & 1.20 & 1.10 & 0.70 & 1.20 \\
\hline Bebidas & 0.00 & 0.80 & 0.0 & 0.60 & 0.50 & 0.20 & 0.60 \\
\hline $\begin{array}{l}\text { Centros } \\
\text { Comerciales }\end{array}$ & 0.00 & 0.10 & 0.10 & 0.10 & 0.10 & 0.00 & 0.10 \\
\hline Consumo & 0.00 & 0.30 & 0.30 & 0.30 & 0.50 & 0.10 & 0.30 \\
\hline $\begin{array}{l}\text { Empresas } \\
\text { productivas } \\
\text { del estado }\end{array}$ & 0.00 & 3.90 & 2.80 & 2.50 & 2.20 & 1.90 & 2.50 \\
\hline Estados & 0.00 & 0.80 & 0.40 & 0.30 & 0.20 & 0.40 & 0.30 \\
\hline Europesos & 0.00 & 2.90 & 2.80 & 2.60 & 2.40 & 0.60 & 2.50 \\
\hline $\begin{array}{l}\text { Grupos } \\
\text { Industriales }\end{array}$ & 0.00 & 0.80 & 0.60 & 0.60 & 0.40 & 0.00 & 0.50 \\
\hline Infraestructura & 0.00 & 5.10 & 4.00 & 3.80 & 4.50 & 1.20 & 3.90 \\
\hline Inmobiliario & 0.00 & 0.00 & 0.00 & 0.10 & 0.10 & 0.00 & 0.10 \\
\hline Otros & 0.00 & 1.20 & 1.00 & 0.90 & 0.80 & 0.40 & 0.90 \\
\hline Papel & 0.00 & 0.10 & 0.10 & 0.10 & 0.00 & 0.00 & 0.10 \\
\hline $\begin{array}{l}\text { Servicios } \\
\text { Financieros }\end{array}$ & 0.00 & 0.40 & 0.40 & 0.40 & 0.50 & 0.10 & 0.40 \\
\hline Telecom & 0.00 & 1.20 & 1.20 & 1.00 & 0.90 & 0.10 & 1.00 \\
\hline Transporte & 0.00 & 0.40 & 0.50 & 0.50 & 0.60 & 0.00 & 0.50 \\
\hline Vivienda & 0.00 & 2.10 & 1.30 & 1.20 & 1.10 & 0.90 & 1.20 \\
\hline Estructurados & 0.00 & 0.90 & 6.00 & 7.30 & 6.40 & 0.10 & 6.10 \\
\hline FIBRAS & 0.00 & 1.70 & 2.50 & 2.40 & 2.50 & 1.80 & 2.40 \\
\hline Renta Variable & 0.00 & 2.20 & 5.20 & 6.00 & 8.20 & 4.40 & 5.90 \\
\hline $\begin{array}{l}\text { Deuda } \\
\text { Internacional }\end{array}$ & 0.00 & 2.00 & 1.30 & 1.10 & 1.40 & 0.00 & 1.20 \\
\hline $\begin{array}{l}\text { Renta Variable } \\
\text { Internacional }\end{array}$ & 0.00 & 3.60 & 10.90 & 12.50 & 15.60 & 13.10 & 12.10 \\
\hline Mercancías & 0.00 & 0.00 & 0.20 & 0.30 & 0.30 & 0.40 & 0.20 \\
\hline Total & 100.00 & 100.00 & 100.00 & 100.00 & 100.00 & 100.00 & 100.00 \\
\hline
\end{tabular}

Cifras al cierre de junio 2019.

Fuente: CONSAR (2019c). 
Marco jurídico de las pensiones mexicanas: análisis empírico mediante productos ... / H.A. OLIVARES AGUAYO et al.

Tabla 16: Diferencias monetarias en el primer y segundo trimestre de 2019 en SIEFORES (cifras en millones de pesos)

\begin{tabular}{|c|c|c|c|c|c|c|c|}
\hline Concepto & SB0 & SB1 & SB2 & SB3 & SB4 & SA & Total \\
\hline $\begin{array}{l}\text { Diferencias } \\
\text { reales }\end{array}$ & 102.58 & -867.74 & $47,524.80$ & $49,223.82$ & $54,376.77$ & $5,313.02$ & $155,673.25$ \\
\hline $\begin{array}{l}\text { Diferencias } \\
\text { Materiales }\end{array}$ & $7,879.96$ & $10,458.30$ & $117,054.35$ & $92,491.80$ & $74,764.62$ & $7,681.60$ & $310,330.62$ \\
\hline $\begin{array}{l}\text { Diferencias } \\
\text { Industrial }\end{array}$ & 762.34 & $1,011.78$ & $11,324.45$ & $8,948.15$ & $7,233.10$ & 743.15 & $30,022.98$ \\
\hline $\begin{array}{l}\text { Diferencias } \\
\text { Financiero }\end{array}$ & $1,659.43$ & $2,202.39$ & $24,650.35$ & $19,477.76$ & $15,744.59$ & $1,617.65$ & $65,352.16$ \\
\hline $\begin{array}{l}\text { Diferencias } \\
\text { Consumo } \\
\text { Frecuente }\end{array}$ & 873.66 & $1,159.53$ & $12,978.09$ & $10,254.79$ & $8,289.31$ & 851.67 & $34,407.05$ \\
\hline
\end{tabular}

Fuente: elaboración propia mediante Excel 2016.

En la Tabla 16 se puede apreciar que para todos los tipos de SIEFORES la inversión en el Sector Materiales fue la que mostró mejores resultados. Pues, las ganancias respecto al trimestre anterior son las más elevadas, lo anterior se resume en una ganancia total de 310,330.62 millones de pesos mexicanos, que es prácticamente el doble que lo mostrado por la CONSAR mediante la diversificación de activos. Asimismo, en los casos mostrados de los otros sectores, se observa que para el caso de la SB0 y SB1 siempre se obtuvieron mayores ganancias a las mostradas por la CONSAR. Y para las otras SIEFORES no se mostraron pérdidas. Como sí la tuvo realmente la CONSAR en la SB1 por casi mil millones de pesos, lo cual es preocupante porque justo en esta SIEFORE están invertidos los recursos de los trabajadores de 60 años o más y de seguir empleando las mismas metodologías se pudiera extrapolar esa cantidad trimestre a trimestre.

\section{CONCLUSIONES Y REFLEXIONES FINALES}

El artículo 18 de la Ley de la CONSAR establece que las administradoras deberán efectuar todas las gestiones que sean necesarias, para la obtención de una adecuada rentabilidad y seguridad en las inversiones de las sociedades de inversión que administren. En cumplimiento de sus funciones, atenderán exclusivamente al interés de los trabajadores y asegurarán que todas las operaciones que efectúen para la inversión de los recursos de dichos trabajadores se realicen con ese objetivo.

Es importante no perder de vista que la Seguridad Social presente y futura del trabajador es un Derecho Humano, en gran medida de ello depende 
su calidad de vida, por lo que, las administradoras no solo deben buscar los mejores rendimientos, están obligadas a hacerlo, ponderando seguridad y rendimiento, con base en el marco de la Ley, pero en ese margen de maniobra deben comprobar que están realizando las mejores inversiones. Por lo que con fundamento en los principios de protección de la persona derivados de las obligaciones constitucionales de protección de derechos humanos, las administradoras deben actuar bajo esta premisa.

Como se observó en los antecedentes de la creación del sistema de capitalización individual, uno de los argumentos centrales para la aprobación fue el desequilibrio actuarial propiciado por los cambios en el número de pensionados y trabajadores activos, aunado a que se sostenía que las AFORES podrían hacer que los ahorros de los trabajadores tuvieran grandes beneficios derivados de las inversiones, de ahí que la necesidad de encontrar mecanismos para lograr el mayor beneficio no debe restringirse, sino que deben adoptarse mecanismos que aumenten los rendimientos de los ahorros, tal como se muestra en la alternativa propuesta en esta investigación mediante productos financieros "estructurados" Call Spread y Put Spread, ya que estos productos al ser de capital garantizado, garantizan la no pérdida de los recursos iniciales trimestrales; se ha demostrado que pueden existir también ganancias mucho mayores a las reportadas por la CONSAR al menos para el cierre del primer y segundo trimestre de 2019; como se mostró en el subyacente Sector Materiales.

Invertir la totalidad de los recursos en productos financieros "estructurados" además de garantizar la no pérdida de los recursos del trabajador también puede generar una mayor cantidad de ganancias para los trabajadores en cualquier tipo de SIEFORE que se encuentren, pues, actualmente el porcentaje de inversión en este tipo de productos es muy pequeño, es decir, se encuentra por debajo de los límites establecidos; y se opta mejor por diversificar los recursos en demasiados productos financieros, lo anterior pone en riesgo las pérdidas potenciales que pueden tener los recursos de los trabajadores como actualmente está ocurriendo, principalmente en la SB1.

Esta investigación demuestra empíricamente, al menos para el cierre de los dos primeros trimestres de 2019, que la actual forma de inversión de los recursos de los trabajadores es menos viable que una inversión total de los recursos en productos financieros "estructurados". Particularmente, se observan afectaciones a las SB0 y SB1 correspondientes a la población de 60 años o más. Por lo que se concluye que el uso de "estructurados" es viable y se recomienda incrementar los porcentajes de inversión en este tipo de 
productos financieros que actualmente realiza la CONSAR para cada tipo de SIEFORE, pues en esta investigación se muestra que, inclusive, la SB0 registró pérdidas trimestrales al cierre del primer trimestre de 2019.

No omitimos preciar que este trabajo tiene como limitación que los resultados obtenidos no consideran los ingresos que tienen los trabajadores durante el trimestre; es decir únicamente las ganancias mostradas en todos los casos son producto de los rendimientos generados por el producto financiero Estructurado; sin embargo, la información mostrada por la CONSAR cada trimestre sí considera dicha información.

\section{REFERENCIAS BIBLIOGRÁFICAS}

Aguiló, G. y Echeverría, C., 2020, Análisis del Sistema de Pensiones Chileno: Orígenes, Evolución, Propuestas Existentes y una Propuesta Innovadora. Universidad de Chile. Disponible en http://repositorio.uchile.cl/bitstream/ handle/2250/177182/Analisis-del-sistema-de-pensiones-chileno-origenes-evolucion-propuestas-existentes-y-una-propuesta-innovadora.pdf? sequence $=1 \&$ isAllowed=y. Fecha de consulta: 03/01/2021.

Amaro, L.C., y Alfonso, L. E., 2018, “¿Cuáles son los efectos del envejecimiento de la población en los sistemas de pensiones de Brasil, España y Francia?", en Revista Brasileira de Estudios de Populacao, vol. 35, núm. 2.

Arce, C. G., 1972, De los seguros sociales a la seguridad social. México: Porrúa. Black, F. y Scholes, M., 1973, "The Pricing of Options and Corporate Liabilities", in The Journal of Political Economy, vol. 81, No. 3, pp. 637-654.

BMV, 2019, Índices Sectoriales Invertibles. Disponible en bmv.com.mx/es/índices/invertibles/. Fecha de consulta: 29/11/2019.

CONSAR, 2019a, Las comisiones que cobran las AFORE importan. Gobierno de México.

CONSAR, 2019b, Primer trimestre 2019. Disponible en www.gob.mx/consar. Fecha de consulta: 10/04/2019.

CONSAR, 2019c, Segundo trimestre 2019, Disponible en: www.gob.mx/consar. Fecha de consulta: 20/07/2019.

CONSAR, 2018, Cuarto trimestre 2018, Disponible en www.gob.mx/consar. Fecha de consulta: 09/02/2021.

Constitución Política de los Estados Unidos Mexicanos, 2020, Diario Oficial de la Federación. Congreso de la Unión, México.

Cruz, M., 1997, Con proyecto de decreto que reforma diversas disposiciones de la Ley de los Sistemas de Ahorro para el Retiro; y de la Ley del Instituto del Fondo Nacional de la Vivienda para los Trabajadores, presentada por el diputado Amado Jesús Cruz Malpica. Gaceta parlamentaria Cámara de Diputados. México. 
De Buen, L. N., 1997, Instituciones de Derecho del Trabajo y la Seguridad Social. México: Universidad NacionalAutónoma de México.

Dopierata, L. y Mosionek, M., 2021, “Gestión de fondos de pensiones, rendimiento de las inversiones y pastoreo en el contexto de cambios regulatorios: nueva evidencia del sistema de pensiones polaco”, en Riesgos, vol. 9, núm.1, pp.1-19.

Farfán, G., 2017, "México: la constitución de 1917 y las reformas de los sistemas de pensiones", en Revista Latinoamericana de Derecho Social, vol. 24, núm.1, pp.3-37

García, M. Y., 2015, “Consideraciones sobre la evolución y retos del Sistema de Ahorro para el Retiro", en Fundación de Estudios Financieros, vol. 4, núm. 1.

Kim, D., Koedel, C., y Xiang, P. B., 2021, "La compensación entre los costos de las pensiones y los gastos salariales en el sector público", en Revista de economía y finanzas de pensiones, vol. 20, núm. 1, pp. 151-168.

Koh, B. S., Mitchell, O. S., y Fong, J. H., 2021, “Confianza y preparación para la jubilación: evidencia de Singapur", en Revista de economía del envejecimiento, vol. 18 , núm. 1.

Kyriakow, I., Mousavi, P., Nielsen, J., y Scholz M., 2021, "Pronóstico de puntos de referencia de rentabilidad de acciones a largo plazo a través del aprendizaje automático", en Canales de Investigación Operativa, vol. 297, núm. 2, pp. 221-240.

Lara, C. y Silva F., 2014, "El Sistema de Pensiones de Chile en la Encrucijada: reformando la Gran Reforma de 2008", en Textos and Contextos Porto Alegre, vol. 13, núm. 1, pp. 113-127.

Ley de los Sistemas de Ahorro para el Retiro, 2014, Diario Oficial de la Federación, Congreso de la Unión, México.

Ley del Seguro Social, 2019, Diario Oficial de la Federación, Congreso de la Unión, México.

López Herrera, Francisco, Martínez Preece, Marissa del Rosario, y Santillán Salgado, Roberto Joaquín, 2020, "Regime-Switching in the Volatility of Mexican Pension Fund Returns”, en Periz Ortiz, Marta; Álvarez García, José; Domínguez Fabián, Inmaculada; y Devolder, Pierre (editores), Economic Challenges of Pension Systems: A Sustainability and International Management Perspective, vol. 1, Springer: Suiza. pp. 397-425.

López, F., y Walker, E., 2021, "Desempeño de la inversión, regulación e incentivos: el caso de los fondos de pensiones chilenos", en Revista de economía y finanzas de pensiones, vol. 20, núm.1, pp.125-150.

McCarthy, M., Pana, E., y Weinberger, A., 2021, "El papel de los inversores institucionales en las transferencias de riesgo de pensiones", en Revista de Economía y Finanzas, en impresión.

Merton, R., 1973, “Theory of Rational Option Pricing”, en Bell Journal of Economics and Management Science, vol. 4, núm. 1, pp. 141-183. 
Murillo S., y Venegas F., 2011, “Cobertura del sistema de jubilación y factores asociados 1 acceso a una pensión de jubilación en México", en Papeles de Población, vol. 17, núm. 67, pp. 209-230.

Nava, I., y Ham, R., 2006, “Dividendos Demográficos y el Sistema”, en Papeles de Población, CIEAP, Universidad Autónoma del Estado de México, pp. 71-95.

OIT, 2001, Hechos Concretos sobre la Seguridad Social. Ginebra: Organización Internacional del Trabajo.

OIT, 2018, Reversing Pension Privatizations: Rebuilding public pension systems in Eastern Europe and Latin America. International Labour Organization.

Olea, M. A., 1983, Instituciones de Seguridad Social. Madrid: Civitas.

Pérez, R., Morgado I., Pérez, M. y Ramos, O., 2017, “Análisis del actual Sistema de Pensiones en Chile", en Revista Argentina de Investigación en Negocios, vol. 3, núm. 2.

Rodríguez L. R., 2017, “Gestión del riesgo de longevidad en los sistemas públicos de pensiones. Una propuesta para utilizar canjes de longevidad para México", en El Trimestre Económico, vol. 84, núm. 335, pp. 681-706.

Ruiz, M. Á., 1999, Nuevo Derecho de la Seguridad Social. México: Porrúa.

Ruiz-Medina, M. I., Quintero, B. M., y Cuadras, B. D., 2016, "Las pensiones de cesantía-vejes e invalidez de la Ley del IMSS un análisis teórico práctico en trabajadores de la Pymes", en Ra Ximhai, 75.

Sainz, G. R., 2008, Diez años de reformas a la Seguridad Social en México. México: Grupo Parlamentario del PRD Cámara de Diputados Congreso de la Unión LX Legislatura.

Sanchez, L. G., 1987, Derecho Mexicano de la Seguridad Social. México: Cárdenas.

Setter, F., 1982, La Sécurité Sociale et ses Principes. México: Instituto Mexicano del Seguro Social.

Solís, S. F., 2001, "Los sistemas de pensiones en México: la agenda pendiente", en Académico ITAM, 188.

Valmer, 2009, Valoración de Notas Estructuradas. Disponible en http://www. valmercostarica.com/CR/PDF/20110419_Notas_Estructuradas_Costa_Rica.pd, Fecha de consulta 02/11/2019.

Wang, P., Li, Z., y Sun, J., 2021, “Opciones de cartera sólida para un plan de pensiones de $\mathrm{CD}$ con riesgo de inflación y prima de riesgo de reversión a la media en condiciones de ambigüedad", en Mejoramiento, vol. 70, núm. 1, pp.191-224. 


\section{RESUMEN CURRICULAR DE LOS AUTORES}

Héctor Alonso Olivares Aguayo

Profesor-Investigador de Tiempo Completo en la Facultad de Negocios de la Universidad La Salle-México. Es Actuario y Maestro en Finanzas con mención honorífica por la Universidad Nacional Autónoma de México (UNAM). Sus principales líneas de investigación son: i) Pensiones, ii) Portafolios de Inversión, iii) Economía Financiera, iv) Administración de Riesgos Financieros, v) Ingeniería Financiera y vi) Ciencias Actuariales. Ha publicado diversos libros, capítulos de libros y artículos científicos en revistas indizadas especializadas en Economía Financiera. Actualmente en México es miembro del SNI y del Colegio Actuarial Mexicano, y en Colombia de la Asociación Internacional de Riesgo y Actuaría.

Dirección electrónica: hectoralonso.olivares@lasalle.mx

Registro ORCID: https://orcid.org/0000-0003-2609-8627

\section{Ana Laura Medina Conde}

Docente-investigadora de la Universidad Autónoma de Tlaxcala, Posdoctorado CONACYT en Derecho de la Empresa en la Universidad de Zaragoza, España, Doctorado en Derecho en argumentación jurídica con mención honorifica y Maestría en Derecho Fiscal por el Centro de Estudios Jurídico Políticos de la Universidad Autónoma de Tlaxcala, Licenciatura en Derecho Civil. Líneas de investigación: Derecho de la Empresa, Derecho Bursátil, Derecho Fiscal, Derechos Humanos. Es autora y coautora de artículos, libros y capítulos de libros, fue Directora de Investigación del Instituto de Ciencias Sociales y Humanidades de la Universidad Tecnológica de la Mixteca, Catedrática de Posgrado y licenciatura en Derecho Bursátil, Derecho Mercantil, Derecho Fiscal y Argumentación Jurídica, en la Universidad Nacional Autónoma de México, de la Universidad de las Américas Puebla, de la Universidad Anáhuac, investigadora de la Universidad la Salle, México y es Miembro del Sistema Nacional de investigadores nivel I.

Dirección electrónica: analaura.medinaconde@uatx.mx

Registro ORCID: https://orcid.org/0000-0003-4203-4383

\section{Oscar Javier Apáez Pineda}

Profesor-Investigador de la Facultad de Derecho de la Universidad La Sa1le-México. Es Licenciado en Derecho por la Universidad Autónoma del estado de Morelos, especialista en Derecho Social y Maestro en Derecho 
por la UNAM. Doctor en Derecho y Globalización con mención honorífica por la Universidad Autónoma del Estado de Morelos. Ha publicado diversos libros, capítulos de libros y artículos científicos en revistas indizadas especializadas en Derecho. Actualmente es miembro del SNI y del Colegio de Profesores de Derecho del Trabajo de la UNAM, académico de número de la Academia Mexicana de Derecho de la Seguridad Social y miembro honorífico del Panel Disciplinario del Comité Nacional Antidopaje (MEX-NADO). Además, es líder responsable del Grupo de Investigación Desarrollo e Innovación: Estudios sobre Justicia Social, Pobreza y Desigualdad de la Universidad La Salle, México.

Dirección electrónica: oscar.apaez@lasalle.mx

Registro ORCID: https://orcid.org/0000-0003-2865-2740 